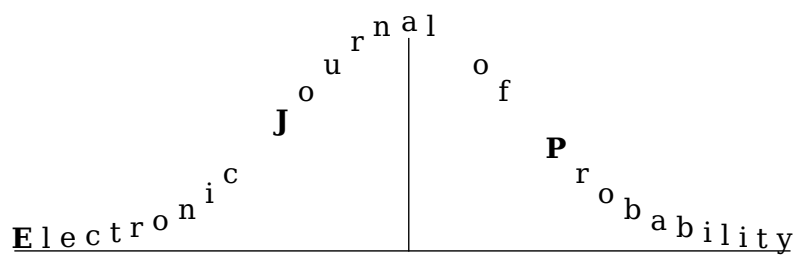

Electron. J. Probab. 24 (2019), no. 38, 1-35.

ISSN: 1083-6489 https://doi.org/10.1214/19-EJP296

\title{
Random walk in cooling random environment: ergodic limits and concentration inequalities
}

\author{
Luca Avena* $\quad$ Yuki Chino ${ }^{\dagger} \quad$ Conrado da Costa ${ }^{\ddagger}$ \\ Frank den Hollander ${ }^{\S}$
}

\begin{abstract}
In previous work by Avena and den Hollander [3], a model of a random walk in a dynamic random environment was proposed where the random environment is resampled from a given law along a given sequence of times. In the regime where the increments of the resampling times diverge, which is referred to as the cooling regime, a weak law of large numbers and certain fluctuation properties were derived under the annealed measure, in dimension one. In the present paper we show that a strong law of large numbers and a quenched large deviation principle hold as well. In the cooling regime, the random walk can be represented as a sum of independent variables, distributed as the increments of a random walk in a static random environment over diverging periods of time. Our proofs require suitable multi-layer decompositions of sums of random variables controlled by moment bounds and concentration estimates. Along the way we derive two results of independent interest, namely, concentration inequalities for the random walk in the static random environment and an ergodic theorem that deals with limits of sums of triangular arrays representing the structure of the cooling regime. We close by discussing our present understanding of homogenisation effects as a function of the cooling scheme, and by hinting at what can be done in higher dimensions. We argue that, while the cooling scheme does not affect the speed in the strong law of large numbers nor the rate function in the large deviation principle, it does affect the fluctuation properties.
\end{abstract}

Keywords: random walk; dynamic random environment; resampling times; law of large numbers; large deviation principle; concentration inequalities.

AMS MSC 2010: 60F05; 60F10; 60G50; 60K37.

Submitted to EJP on October 25, 2018, final version accepted on March 17, 2019.

${ }^{*}$ Leiden University, the Netherlands. E-mail: l . avena@math . leidenuniv. $\mathrm{nl}$

${ }^{\dagger}$ Leiden University, the Netherlands. E-mail: y. chino@math. leidenuniv. nl

${ }^{\ddagger}$ Leiden University, the Netherlands. E-mail: c . freitas . paulo. da. costa@math . leidenuniv. nl

${ }^{\S}$ Leiden University, the Netherlands. E-mail: denhol la@math. leidenuniv.nl 


\section{Introduction, main results and discussion}

Random walk in random environment is a model for a particle moving in an inhomogeneous potential. When the random environment is static this model exhibits striking features. Namely, there are regions where the random walk remains trapped for a long time. The presence of these traps leads to a slow-down of the random walk in comparison to a homogeneous random walk, and may result in anomalous scaling, especially in low dimensions. At present, these slow-down phenomena have been fully understood only in dimension one (see Zeitouni [16] for an overview, and references therein).

The situation where the random environment is dynamic has seen major progress in the last ten years. While the random environment evolves over time, it remains inhomogeneous but dissolves existing traps and creates new traps. Depending on the choice of the dynamics, the random walk behaviour can either be similar to that in the static model or be similar to that in the homogeneous model. Up to now, most dynamic models require strong space-time mixing conditions, guaranteeing negligible trapping effects and resulting in scaling properties similar to those of a homogeneous random walk (see Avena, Blondel and Faggionato [2] for an overview, and references therein).

In Avena and den Hollander [3], a new random walk model was introduced, called Random Walk in Cooling Random Environment (RWCRE). This has a dynamic random environment, but differs from other dynamic models in that it allows for an explicit control of the time mixing in the environment. Namely, at time zero an i.i.d. random environment is generated, and this is fully resampled along an increasing sequence of deterministic times. If the resampling times increase rapidly enough, then we expect to see a behaviour close to that of the static model. Conversely, if the resampling times increase slowly enough, then we expect to see a behaviour that is close to the homogeneous model. Thus, RWCRE allows for different scenarios as a function of the speed of growth of the resampling times. The name "cooling" is used because the static model is sometimes called "frozen".

In order to advance our understanding of RWCRE, we need to acquire detailed knowledge of fluctuations and large deviations for the classical one-dimensional Random Walk in Random Environment (RWRE). Part of this knowledge is available from the literature, but part is not and needs to be developed along the way. A few preliminary results were proved in Avena and den Hollander [3] under the annealed law. In the simplest scenario where the increments of the resampling times stay bounded, which is referred to as the no-cooling regime, full homogenisation takes place, and both a classical Strong Law of Large Numbers (SLLN) and a classical Central Limit Theorem (CLT) hold. Moreover, it was shown that as soon as the increments of the resampling times diverge, which is referred to as the cooling regime, a Weak Law of Large Numbers (WLLN) holds with an asymptotic speed that is the same as for the corresponding RWRE [3, Theorem 1.5]. As far as fluctuations are concerned, for the case where the RWRE is in the so-called Sinai regime (recurrent, subdiffusive, non-standard limit law; see Sinai [14], Kesten [10]), it was shown that RWCRE exhibits Gaussian fluctuations with a scaling that depends on the speed of divergence of the increments of the resampling times [3, Theorem 1.6]. The proof of this fact requires that the convergence to the limit law for the corresponding RWRE is in $L^{p}$ for some $p>2$. In [3, Appendix C] it was shown that the convergence is in $L^{p}$ for all $p>0$.

In the present paper we pursue a more refined investigation of RWCRE. We focus on the cooling regime and aim for a deeper understanding of homogenisation effects. In particular, we derive a SLLN and a quenched Large Deviation Principle (LDP), with a speed and a rate function that are the same as for the corresponding RWRE, no matter how slow or how fast the cooling scheme (Theorems 1.10 and 1.11 below). Both results 
are not unexpected, but at the same time are far from obvious. As we will see, they lead to some subtle surprises, which we discuss below. A crucial ingredient in both proofs is a general limit property we call cooling ergodic theorem, which is needed to control certain variables representing the structure of the cooling regime (Theorem 1.12 below). This theorem not only is a key tool in our proofs, it will also be useful to address other questions not investigated here. To prove the SLLN and the LDP we also need certain concentration inequalities for the corresponding RWRE (Theorem 1.13 below).

Outline. In Section 1.1 we define one-dimensional RWRE and recall some basic facts that are used throughout the paper. In Section 1.2 we define RWCRE. In Section 1.3 we state our four main theorems and provide some insight into their proofs. In Section 1.4 we discuss what is known about RWCRE, explain how the results derived so far relate to each other, and state a number of open problems. The remainder of the paper is devoted to the proofs: Section 2 for the cooling ergodic theorem mentioned above, Section 3 for the concentration inequalities of RWRE, and Section 4 for the SLLN and the LDP of RWCRE.

\subsection{RWRE: some basic facts}

Throughout the paper we use the notation $\mathbb{N}_{0}=\mathbb{N} \cup\{0\}$ with $\mathbb{N}=\{1,2, \ldots\}$. The classical one-dimensional static model is defined as follows. Let $\omega=\{\omega(x): x \in \mathbb{Z}\}$ be an i.i.d. sequence with probability distribution

$$
\mu=\alpha^{\mathbb{Z}}
$$

for some probability distribution $\alpha$ on $(0,1)$. We write $\langle\cdot\rangle$ to denote the expectation w.r.t. $\alpha$.

Definition 1.1 (RWRE). Let $\omega$ be an environment sampled from $\mu$. We call Random Walk in Random Environment the Markov chain $Z=\left(Z_{n}\right)_{n \in \mathbb{N}_{0}}$ with state space $\mathbb{Z}$ and transition probabilities

$$
P^{\omega}\left(Z_{n+1}=x+e \mid Z_{n}=x\right)=\left\{\begin{array}{ll}
\omega(x) & \text { if } e=1, \\
1-\omega(x) & \text { if } e=-1,
\end{array} \quad x \in \mathbb{Z}, n \in \mathbb{N}_{0} .\right.
$$

We denote by $P_{x}^{\omega}(\cdot)$ the quenched law of the Markov chain identified by the transitions in (1.2) starting from $x \in \mathbb{Z}$, and by

$$
P_{x}^{\mu}(\cdot)=\int_{(0,1)^{\mathbb{Z}}} P_{x}^{\omega}(\cdot) \mu(\mathrm{d} \omega)
$$

the corresponding annealed law.

The understanding of one-dimensional RWRE is well developed, both under the quenched and the annealed law. For a general overview, we refer the reader to the lecture notes by Zeitouni [16]. Here we collect some basic facts and definitions that will be needed throughout the paper.

The asymptotic properties of RWRE are controlled by the distribution of the ratio of the transition probabilities to the left and to the right at the origin, i.e.,

$$
\rho=\frac{1-\omega(0)}{\omega(0)} \text {. }
$$

We will impose a uniform ellipticity condition on $\mu$, namely,

$$
\exists \mathfrak{c} \in(0,1): \quad \operatorname{supp}(\alpha) \subset[\mathfrak{c}, 1-\mathfrak{c}] .
$$


Definition 1.2 (Basic environment distribution). We call a probability distribution $\mu$ on $(0,1)^{\mathbb{Z}} \alpha$-basic if (1.1) and (1.5) hold.

The following proposition due to Solomon [15] characterises recurrence versus transience and asymptotic speed. To state the result in a simple form we may assume without loss of generality that

$$
\langle\log \rho\rangle \leq 0 .
$$

The case where $\langle\log \rho\rangle>0$ follows by a reflection argument. Indeed, define $\widetilde{\omega}$ by $\widetilde{\omega}(x)=1-\omega(-x), x \in \mathbb{Z}$. From (1.2) we see that $P_{0}^{\omega}\left(-Z_{n} \in \cdot\right)=P_{0}^{\widetilde{\omega}}\left(Z_{n} \in \cdot\right)$. Therefore, statements for the left of the origin can be obtained from statements for the right of the origin in the reflected environment and so (1.6) is assumed for convenience.

Proposition 1.3 (Recurrence, transience and speed of RWRE [15]). Suppose that $\mu$ is $\alpha$-basic and that (1.6) holds. Then:

- $Z$ is recurrent when $\langle\log \rho\rangle=0$.

- $Z$ is transient to the right when $\langle\log \rho\rangle<0$.

- For $\mu$-a.e. $\omega, P_{0}^{\omega}$ - a.s.,

$$
\lim _{n \rightarrow \infty} \frac{Z_{n}}{n}=v_{\mu}= \begin{cases}0, & \text { if }\langle\rho\rangle \geq 1, \\ \frac{1-\langle\rho\rangle}{1+\langle\rho\rangle}>0, & \text { if }\langle\rho\rangle<1 .\end{cases}
$$

The above proposition shows that the speed of RWRE is a deterministic function of $\mu$ (or of $\alpha$; recall (1.1)). Note that for $\alpha$ such that $\langle\log \rho\rangle\langle 0$ and $\langle\rho\rangle \geq 1$, the random walk is transient to the right with zero speed. In this regime $Z$ diverges, but only sublinearly due to the presence of traps, i.e., local regions of the environment pushing the random walk against its global drift.

Similar trapping effects give rise to other anomalous behaviour for fluctuations and large deviations. In order to state the latter, we recall that a family of probability measures $\left(P_{n}\right)_{n \in \mathbb{N}}$ defined on the Borel sigma-algebra of a topological space $(\mathcal{S}, \mathcal{T})$ is said to satisfy the LDP with rate $n$ and with rate function $I: \mathcal{S} \rightarrow[0, \infty]$ when

$$
\begin{array}{ll}
\liminf _{n \rightarrow \infty} \frac{1}{n} \log P_{n}(\mathcal{O}) \geq-\inf _{x \in \mathcal{O}} I(x) & \forall \mathcal{O} \subset \mathcal{S} \text { open }, \\
\limsup _{n \rightarrow \infty} \frac{1}{n} \log P_{n}(\mathcal{C}) \leq-\inf _{x \in \mathcal{C}} I(x) & \forall \mathcal{C} \subset \mathcal{S} \text { closed },
\end{array}
$$

$I$ has compact level sets and $I \not \equiv \infty$ (see e.g. den Hollander [9, Chapter III]). The following proposition due to Greven and den Hollander [8] identifies the LDP for the empirical speed under the quenched law.

Proposition 1.4 (Quenched LDP for RWRE displacements [8]).

Suppose that $\mu$ is $\alpha$-basic. Then, for $\mu$-a.e. $\omega,\left(Z_{n} / n\right)_{n \in \mathbb{N}}$ under $P_{0}^{\omega}$ satisfies the LDP on $\mathbb{R}$ with rate $n$ and with a convex and deterministic rate function $\mathcal{I}=\mathcal{I}_{\mu}$.

See [8] for a representation of $\mathcal{I}$ in terms of random continued fractions and Fig. 2 for the qualitative behaviour of $\mathcal{I}$ on different regimes.

In the sequel we will need refined results about the cumulant generating function of $Z_{n} / n$. For that we need to introduce the hitting times to the right

$$
H_{n}=\inf \left\{m \in \mathbb{N}: Z_{m}=n\right\}, \quad n \in \mathbb{N},
$$

state the weak LDP for $H_{n} / n$, which was derived in Comets, Gantert and Zeitouni [6], and show its relation with the LDP for $Z_{n} / n$. See also den Hollander [9, Chapter VII]. We recall that for the weak LDP the second line in (1.8) is only required to hold for compact sets, and the rate function is only required to be lower semi-continuous. 


\section{Proposition 1.5 (Quenched LDP for RWRE hitting times [6]).}

Suppose that $\mu$ is $\alpha$-basic. Then, for $\mu$-a.e. $\omega,\left(H_{n} / n\right)_{n \in \mathbb{N}}$ under $P_{0}^{\omega}$ satisfies the weak LDP on $\mathbb{R}$ with rate $n$ and with a convex and deterministic weak rate function $\mathcal{J}=\mathcal{J}_{\mu}$ given by (see Fig. 1)

$$
\mathcal{J}(x)=\sup _{\lambda \in \mathbb{R}}\left[\lambda x-\mathcal{J}^{*}(\lambda)\right], \quad x \in \mathbb{R},
$$

where

$$
\mathcal{J}^{*}(\lambda)=\lim _{n \rightarrow \infty} \frac{1}{n} \log E_{0}^{\omega}\left[e^{\lambda H_{n}}\right]=\int_{(0,1)^{\mathbb{Z}}} \mu(\mathrm{d} \omega) \log E_{0}^{\omega}\left[e^{\lambda H_{1}}\right] \quad \omega-\text { a.s., } \lambda \in \mathbb{R} .
$$
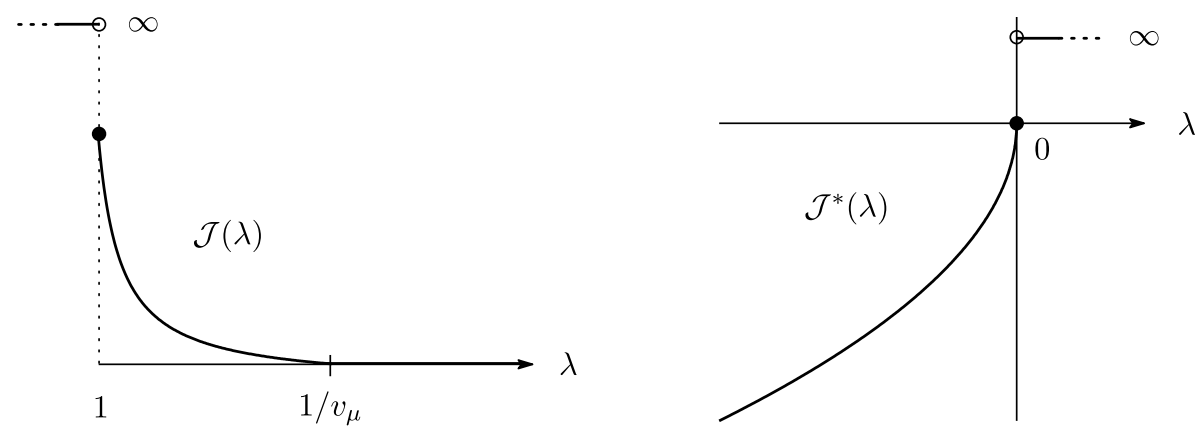

Figure 1: Left: Graph of $\mathcal{J}$, the quenched rate function of RWRE hitting times in (1.10). Right: Graph of $\mathcal{J}^{*}$, the scaled cumulant generating function of RWRE hitting times in (1.11).

For the hitting times to the left, defined by (1.9) with $n \in-\mathbb{N}$, we have the weak rate function $\widetilde{\mathcal{J}}=\widetilde{\mathcal{J}}_{\mu}$ :

$$
\tilde{\mathcal{J}}(x)=\mathcal{J}(x)-\langle\log \rho\rangle, \quad x \in \mathbb{R} .
$$

Moreover, the following relation between $\mathcal{J}$ and $\mathcal{I}$ holds (see [9, Chapter VII]):

$$
\mathcal{I}(x)= \begin{cases}x \mathcal{J}(1 / x), & x \in(0,1], \\ 0, & x=0, \\ (-x) \widetilde{\mathcal{J}}(1 /(-x)), & x \in[-1,0) .\end{cases}
$$

The empirical speed of RWRE also satisfies the LDP under the annealed law.

\section{Proposition 1.6 (Annealed LDP for RWRE displacements [6]).}

Suppose that $\mu$ is $\alpha$-basic. Then $\left(Z_{n} / n\right)_{n \in \mathbb{N}}$ under $P_{0}^{\mu}$ satisfies the LDP on $\mathbb{R}$ with rate $n$ and with a convex rate function $\mathcal{I}^{\text {ann }}=\mathcal{I}_{\mu}^{\text {ann }}$.

As shown in [6], the annealed and the quenched rate function are related through the following variational principle

$$
\mathcal{I}^{\mathrm{ann}}(\theta)=\mathcal{I}_{\mu}^{\mathrm{ann}}(\theta)=\inf _{\nu}\left[\mathcal{I}_{\nu}(\theta)+|\theta| h(\nu \mid \mu)\right]
$$

where $\mathcal{I}_{\nu}$ is the quenched rate function associated with a random environment that has law $\nu, h(\nu \mid \mu)$ denotes the relative entropy of $\nu$ with respect to $\mu$, and the infimum runs over the set of probability measures on $(0,1)^{\mathbb{Z}}$ endowed with the weak topology (see [6] for more details). In particular, $\mathcal{I}^{\text {ann }}$ is qualitatively similar to $\mathcal{I}$ in Fig. 2 , in the sense that $\mathcal{I}^{\text {ann }}$ is strictly decreasing on $[-1,0]$, zero on $\left[0, v_{\mu}\right]$, and strictly increasing on $\left[v_{\mu}, 1\right]$. 

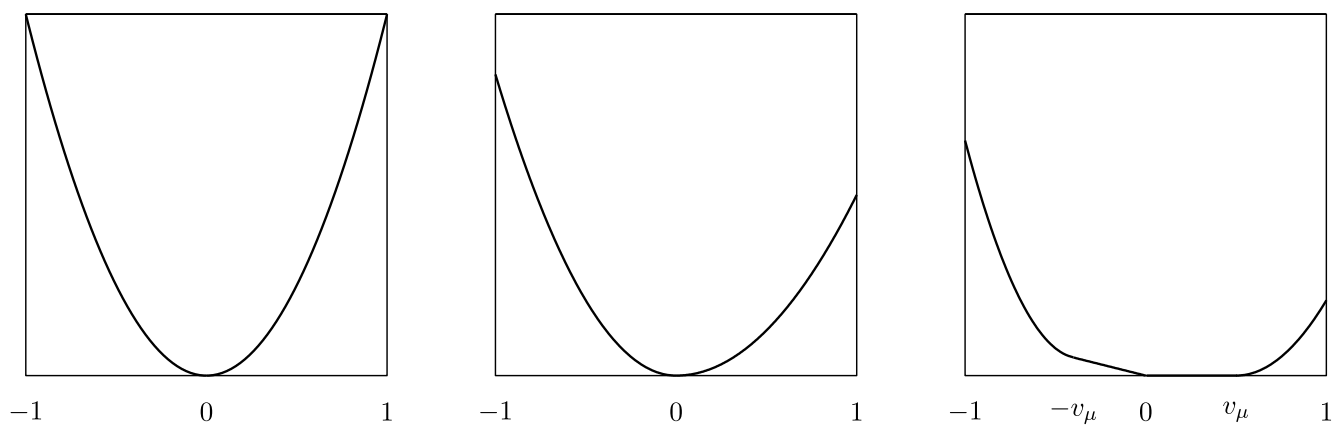

Figure 2: Graph of $\mathcal{I}$, the quenched rate function of RWRE displacements in (1.13). Three cases are shown from left to right: recurrent, transient with zero speed, transient with positive speed.

The presence of the flat piece $\left[0, v_{\mu}\right]$ in the positive speed case makes our analysis more delicate, and we will need the following large deviation bound characterising the right decay when zooming in on the flat piece:

Proposition 1.7 (Annealed LDP bound away from $v_{\mu}[7]$ ).

Suppose that $\mu$ is $\alpha$-basic. Then, for any closed set $\mathcal{C}$ such that $v_{\mu} \notin \mathcal{C}$,

$$
\limsup _{n \rightarrow \infty} \frac{1}{\log n} \log P_{0}^{\mu}\left(\frac{Z_{n}}{n} \in \mathcal{C}\right)<0 .
$$

\subsection{RWCRE: refreshing times}

The cooling random environment is the space-time random environment built by partitioning $\mathbb{N}_{0}$, and assigning independently to each piece an environment sampled from $\mu$ in (1.1) (see Fig. 3). Formally, let $\tau: \mathbb{N}_{0} \rightarrow \mathbb{R}_{+}$be a strictly increasing function with $\tau(0)=0$, referred to as the cooling map. The cooling map determines a sequence of refreshing times $(\tau(k))_{k \in \mathbb{N}_{0}}$ that we use to construct the dynamic random environment.

Definition 1.8 (Cooling Random Environment). Given a cooling map $\tau$, let $\Omega=$ $\left(\omega_{k}\right)_{k \in \mathbb{N}}$ be an i.i.d. sequence of random variables with law $\mu$ in (1.1). The cooling random environment is built from the pair $(\Omega, \tau)$ by assigning the environment $\omega_{k}$ to the $k$-th interval $I_{k}$ defined by

$$
I_{k}=[\tau(k-1), \tau(k)), \quad k \in \mathbb{N} .
$$

In the present paper we consider the cooling regime, i.e., we consider $\tau$ such that the length of $I_{k}$ in (1.16) diverges:

$$
T_{k}=\tau(k)-\tau(k-1), \quad \lim _{k \rightarrow \infty} T_{k}=\infty .
$$

The role of this assumption is clarified in Section 1.4.

Definition 1.9 (RWCRE). Let $\tau$ be a cooling map and $\Omega$ an environment sequence sampled from $\mu^{\mathbb{N}}$. We call Random Walk in Cooling Random Environment the Markov chain $X=\left(X_{n}\right)_{n \in \mathbb{N}_{0}}$ with state space $\mathbb{Z}$ and transition probabilities

$$
P^{\Omega, \tau}\left(X_{n+1}=x+e \mid X_{n}=x\right)=\left\{\begin{array}{ll}
\omega_{\ell(n)}(x), & e=1, \\
1-\omega_{\ell(n)}(x), & e=-1,
\end{array} \quad x \in \mathbb{Z}, n \in \mathbb{N}_{0},\right.
$$

where

$$
\ell(n)=\inf \left\{k \in \mathbb{N}_{0}: \tau(k)>n\right\}
$$


RWCRE: ergodic limits and concentration inequalities

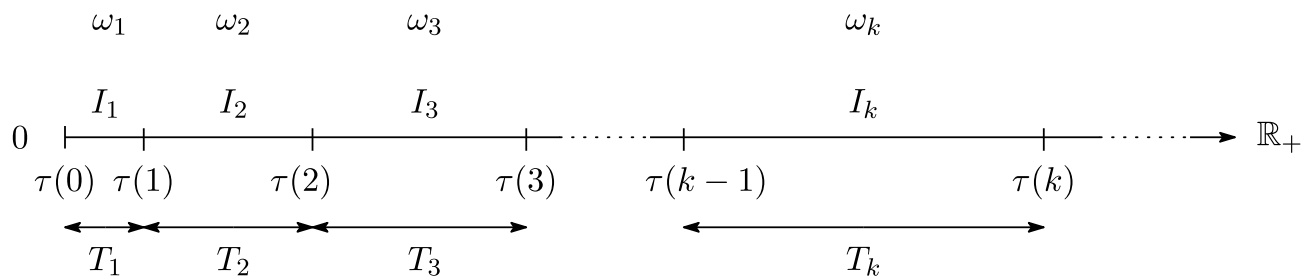

Figure 3: Structure of the cooling random environment $(\Omega, \tau)$.

is the index of the interval $n$ belongs to. Similarly to Definition 1.1 , we denote by

$$
P_{x}^{\Omega, \tau}(\cdot) \quad \text { and } \quad P_{x}^{\mu, \tau}(\cdot)=\int_{\left[(0,1)^{\mathbb{Z}}\right]^{\mathbb{N}}} P_{x}^{\Omega, \tau}(\cdot) \mu^{\mathbb{N}}(\mathrm{d} \Omega),
$$

the corresponding quenched and annealed laws, respectively.

In words, RWCRE moves according to a given environment sampled from $\mu$, until the next refreshing time $\tau(k)$, when a new environment is sampled from $\mu$. Equivalently, the random walk trajectory is independent across the intervals, and during each interval $I_{k}$ moves like a RWRE in the environment $\omega_{k}$. In view of assumption (1.17), the environment is resampled along a diverging sequence of time increments. Our goal is to understand in what way this makes RWCRE behave similarly as RWRE (see Section 1.4 below).

The position $X_{n}$ of RWCRE admits the following key decomposition into pieces of RWRE. Define the refreshed increments and the boundary increment as

$$
Y_{k}=X_{\tau(k)}-X_{\tau(k-1)}, \quad k \in \mathbb{N}, \quad \bar{Y}^{n}=X_{n}-X_{\tau(\ell(n)-1)},
$$

and the running time at the boundary as

$$
\bar{T}^{n}=n-\tau(\ell(n)-1) .
$$

Note that, by (1.17),

$$
\sum_{k=1}^{\ell(n)-1} T_{k}+\bar{T}^{n}=n .
$$

By construction, we can write $X_{n}$ as the sum

$$
X_{n}=\sum_{k=1}^{\ell(n)-1} Y_{k}+\bar{Y}^{n}, \quad n \in \mathbb{N}_{0} .
$$

This decomposition shows that, in order to analyse $X$, we must analyse the vector

$$
\left(Y_{1}, \cdots, Y_{\ell(n)-1}, \bar{Y}^{n}\right)
$$

consisting of independent components, each distributed as an increment of $Z$ (defined in Section 1.1) over a given time length determined by $\tau$ and $n$. Fig. 4 illustrates this piece-wise decomposition of $X_{n}$. More precisely, for any measurable function $f: \mathbb{Z} \rightarrow \mathbb{R}$, any $\Omega$ sampled from $\mu^{\mathbb{N}}$ and any $\tau$,

$$
E_{0}^{\Omega, \tau}\left[f\left(Y_{k}\right)\right]=E_{0}^{\omega_{k}}\left[f\left(Z_{T_{k}}\right)\right], \quad E_{0}^{\Omega, \tau}\left[f\left(\bar{Y}^{n}\right)\right]=E_{0}^{\omega_{\ell(n)}}\left[f\left(Z_{\bar{T}^{n}}\right)\right] .
$$


RWCRE: ergodic limits and concentration inequalities

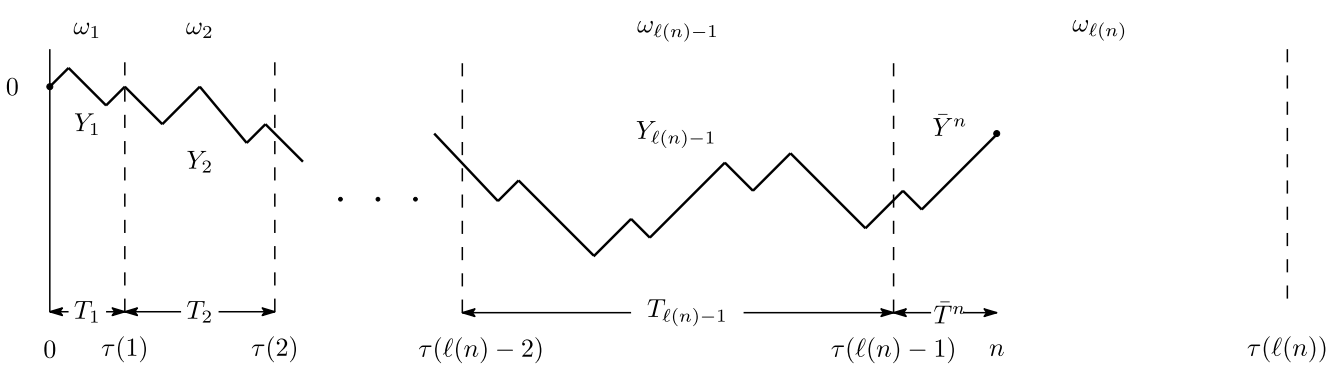

Figure 4: The decomposition of RWCRE in pieces of RWRE as presented in (1.24).

\subsection{Main results}

We can now state our main results for the asymptotic behaviour of RWCRE.

Theorem 1.10 (SLLN for RWCRE displacements).

Suppose that $\mu$ is $\alpha$-basic and that $\tau$ satisfies (1.17). Then, for $\mu^{\mathbb{N}}$ - a.e. $\Omega$,

$$
\lim _{n \rightarrow \infty} \frac{X_{n}}{n}=v_{\mu} \quad P_{0}^{\Omega, \tau}-\text { a.s. }
$$

with $v_{\mu}$ as in (1.7).

\section{Theorem 1.11 (Quenched LDP for RWCRE displacements).}

Suppose that $\mu$ is $\alpha$-basic and that $\tau$ satisfies (1.17). Then, for $\mu^{\mathbb{N}}$-a.e. $\Omega,\left(X_{n} / n\right)_{n \in \mathbb{N}}$ under $P_{0}^{\Omega, \tau}$ satisfies the $L D P$ on $\mathbb{R}$ with rate $n$ and with the same rate function $\mathcal{I}=\mathcal{I}_{\mu}$ as in Proposition 1.4 .

Both theorems will be discussed in Section 1.4 and will be proved in Section 4. Their derivation will be based on the following general convergence statement tailored to RWCRE.

Theorem 1.12 (Cooling Ergodic Theorem).

Let $\left(\psi_{n}^{(k)}\right)_{n, k \in \mathbb{N}}$ be an array of real-valued random variables with law $\mathbb{P}$. Assume that:

(A1) For all $k, k^{\prime} \in \mathbb{N}$ with $k \neq k^{\prime},\left(\psi_{n}^{(k)}\right)_{n \in \mathbb{N}}$ and $\left(\psi_{n}^{\left(k^{\prime}\right)}\right)_{n \in \mathbb{N}}$ are independent.

(A2) There exists a $C \in(0, \infty)$ such that

$$
\sup _{k, n \in \mathbb{N}}\left|n^{-1} \psi_{n}^{(k)}\right|<C \quad \mathbb{P}-\text { a.s. }
$$

(A3) There exist $L \in \mathbb{R}$ and $\delta>1$ such that for all $\varepsilon>0$ there exists a $C^{\prime}=C^{\prime}(\varepsilon) \in(0, \infty)$ such that

$$
\sup _{k \in \mathbb{N}} \mathbb{P}\left(\left|\frac{\psi_{n}^{(k)}}{n}-L\right|>\varepsilon\right)<\frac{C^{\prime}}{n^{\delta}}, \quad \forall n \in \mathbb{N} .
$$

Then, for any cooling map $\tau$ satisfying (1.17),

$$
\lim _{n \rightarrow \infty} \frac{1}{n}\left(\sum_{k=1}^{\ell(n)-1} \psi_{T_{k}}^{(k)}+\psi_{\bar{T}^{n}}^{(\ell(n))}\right)=L \quad \mathbb{P}-\text { a.s. }
$$

with $\ell(n)$ as in (1.19), $T_{k}$ as in (1.17), and $\bar{T}^{n}$ as in (1.22). Furthermore, (1.30) remains valid if (1.29) holds with $\delta \in(0,1]$ and, in addition to (A1) and (A2), there exists a $C \in(0, \infty)$ such that

$$
\sup _{k, n \in \mathbb{N}}\left|\psi_{n+1}^{(k)}-\psi_{n}^{(k)}\right| \leq C \quad \mathbb{P}-\text { a.s. }
$$


Theorem 1.12 is useful for controlling limits of sums of the form appearing in (1.30). Its proof is presented in Section 2 and is based on moments bounds and concentration estimates, applied to a further decomposition into what we call refreshed, boundary and deterministic terms, respectively. Theorem 1.12 is a key ingredient in our paper.

To check (1.29) is a challenge. In itself, (1.29) is only a mild decay requirement, but it forces us to derive concentration inequalities for RWRE, which is a non-trivial task. For the SLLN in Theorem 1.10, the required concentration inequalities are already at our disposal, since they are encoded in the annealed large deviation bound recalled in Proposition 1.7. However, for Theorem 1.11 concentration results for RWRE are needed which, to the best of our knowledge, are not available in the literature. Therefore we state in the following theorem such results, which are of independent interest.

Since the nearest-neighbour walk is not aperiodic, for parity reasons and notational ease we denote by $[n x]$ the lower integer part of $x$, and by $[[n x]]$ either $[n x]$ or $[n x]+1$ depending on the parity of $n$, so that $[[n x]]$ is even/odd when $n$ is even/odd.

Theorem 1.13 (Concentration for RWRE).

Suppose that $\mu$ is $\alpha$-basic. Then, for any $\lambda \in \mathbb{R}, \delta \in(0,1)$ and $\varepsilon>0$ there are $C, c \in(0, \infty)$ (depending on $\mu, \lambda, \delta, \varepsilon$ ) such that

$$
\mu\left(\omega:\left|\frac{1}{n} \log E_{0}^{\omega}\left[e^{\lambda H_{n}}\right]-\mathcal{J}^{*}(\lambda)\right|>\varepsilon\right) \leq C e^{-c n^{1-\delta}}, \quad \forall n \in \mathbb{N},
$$

where $\mathcal{J}^{*}$ is the Legendre transform of the rate function $\mathcal{J}$ in Proposition 1.5,

$$
\mu\left(\omega:\left|\frac{1}{n} \log E_{0}^{\omega}\left[e^{\lambda Z_{n}}\right]-\mathcal{I}^{*}(\lambda)\right|>\varepsilon\right) \leq C e^{-c n^{1-\delta}}, \quad \forall n \in \mathbb{N},
$$

with $\mathcal{I}^{*}$ the Legendre transform of the rate function $\mathcal{I}$ in Proposition 1.4, and

$$
\mu\left(\omega: \sup _{x \in[-1,1]}\left|\frac{1}{n} \log P_{0}^{\omega}\left(Z_{n}=[[n x]]\right)+\mathcal{I}(x)\right|>\varepsilon\right) \leq C e^{-c n^{1-\delta}}, \quad \forall n \in \mathbb{N} .
$$

Note that if $\lambda>0$, then both $E_{0}^{\omega}\left[e^{\lambda H_{n}}\right]$ and $\mathcal{J}^{*}(\lambda)$ are infinite. The proof of Theorem 1.13 is given in Section 3.

\subsection{Discussion}

No-cooling: bounded time increments. The regime where assumption (1.17) does not hold and the increments $T_{k}$ in (1.17) are of order one has been investigated in [3]. Due to the fast resampling, no trapping effects enter the game and full homogenisation takes place. In fact, the decomposition in (1.24) gives us a sum of almost i.i.d. random variables and the resulting behaviour is as if $X$ were a homogeneous Markov chain: [3, Theorem 1.4] shows a corresponding classical SLLN and classical CLT under the annealed law.

Weak and strong law of large numbers. Theorem 1.10 states that, as soon as the cooling is effective, i.e., assumption (1.17) is in force, the asymptotic speed exists a.s., is deterministic and is equal to the one for RWRE. The same statement has been derived in weak form in [3, Theorem 1.5]. The strong form presented here requires a much more involved proof, based on RWRE concentration inequalities. In fact, Theorem 1.10 is far from trivial because the cooling map allows for fluctuations that could in principle hamper the almost sure convergence. As the proof reveals, the fact that this is not the case comes from a non-trivial averaging due to the cooling resampling mechanism. Roughly speaking, the slower the cooling, the stronger are the fluctuations of the constituent pieces in the sum in (1.24), but these fluctuations average out, as will be shown with the help of the moments bounds and the concentration estimates mentioned earlier. 
Large deviations and fluctuations. As soon as the increments between the resampling times diverge, the rate function in the LDP for RWCRE in Theorem 1.11 is the same as for RWRE. In words, the cost to deviate from the typical speed is determined by the trapping in a fixed environment and the resampling has no further homogenising effect. This is true when we look on an exponential scale, but we may expect RWRE and RWCRE to show different large deviation behaviour when we zoom in on the flat piece $\left[0, v_{\mu}\right]$ when $v_{\mu}>0$ (see Fig. 2). Theorem 1.11 deserves further comments because, when we look at fluctuations, RWRE and RWCRE actually give rise to different scaling limits. This has been proved for recurrent RWRE, which exhibits non-standard fluctuations after scaling by $\log ^{2} n$. Indeed, [3, Theorem 1.6] shows that, for certain $\tau^{\prime}$ 's under the annealed law, RWCRE exhibits Gaussian fluctuations after scaling by a factor that grows faster than $\log ^{2} n$ and depends on the cooling map $\tau$. Similar scenarios, and even the presence of a crossover, have been conjectured to hold for RWCRE in other regimes (see Table 1). This may all sound paradoxical, because it is folklore to expect that the zeros of the rate function in an LDP encode information on the order of the fluctuations. However, the latter is only true when the rate function is smooth near its zeros. This is not the case for the rate functions in Fig. 2, and so the paradox is explained.

Relaxing the i.i.d. assumption on $\mu$. It is worth mentioning that the i.i.d. assumption on $\mu$ made in (1.1) can in principle be relaxed to the assumption that $\mu$ is stationary and ergodic with respect to translations. The reader is invited to check that all the steps in the proofs below work in this more general setting. On the other hand, we will make use of certain known properties of RWRE some of which require further technical assumptions to guarantee local product structure (see e.g. [16, Theorem 2.4.3, p. 236] for the extension of Proposition 1.7).

Higher dimensions. RWRE in higher dimensions is much more involved. For instance, the SLLN has been proved only under certain ballisticity conditions. It can be shown that, for RWCRE in the cooling regime, the SLLN in Theorem 1.10 carries over to higher dimensions under precisely the same ballisticity conditions, as a consequence of the Cooling Ergodic Theorem in Theorem 1.12. The LDP in Theorem 1.11 does not automatically carry over because it is based on Theorem 1.13 and an inversion argument, in which we pass from hitting times to RW displacements. This argument exploits the one-dimensional setup, but might in principle be extended to higher dimensions.

Comparison and open problems. We conclude by summarising our present understanding of RWCRE based on the results derived here and in [3]. Let us stress again that the RWCRE model can be seen as a model that interpolates between the classical static model (i.e., $\tau(1)=\infty$ ) and the model with i.i.d. resamplings every unit of time (i.e., $\tau(n)=n$ ). The latter reduces to a homogeneous nearest-neighbour random walk under the annealed measure, but even under the quenched law the independent space-time structure leads to a strong homogenising scenario for which e.g. a classical CLT holds (see e.g. Boldrighini, Minlos and Pellegrinotti [4]). The interesting features therefore appear as we explore different cooling regimes, which allow for a competition between the effect of traps in the static environment and the effect of homogenisation coming from the resampling. Table 1 gives a qualitative comparison for RWRE, RWCRE and standard homogeneous nearest-neighbour random walk, abbreviated as RW. In view of the discussion above, the no-cooling regime $\tau(n) \sim n$ is in the same "universality class" as homogeneous random walk, which is why it is put in the same column as RW. 
RWCRE: ergodic limits and concentration inequalities

\begin{tabular}{|c|c|c|c|}
\hline Model & RW $\simeq$ No-Cooling & RWCRE & RWRE \\
\hline Medium & Homogeneous & Cooling & Static \\
\hline \hline Recurrence & local drift $=0$ & global, depending on $(\tau, \mu)$ ? & $\langle\log \rho\rangle=0$, global \\
\hline Speed & local drift & $v_{\mu}$ (non-local) & $v_{\mu}$ (non-local) \\
\hline LDP rate $n$ & Cramér-analytic rate fn & non-analytic rate fn $\mathcal{I}$ & non-analytic rate fn $\mathcal{I}$ \\
\hline Fluctuations & & $\log \log \tau(n)=o(n):$ Gaussian & Sinai-Kesten \\
$\langle\log \rho\rangle=0$ & \multirow{2}{*}{ CLT } & else: Sinai-Kesten? & scale: $\log ^{2} n$ \\
\cline { 3 - 4 } Fluctuations & & ??? & Kesten-Kozlov-Spitzer \\
$\langle\log \rho\rangle<$ & & & $s<2$ stable law \\
0 & & & $s$ CLT \\
\hline
\end{tabular}

Table 1: Comparison among standard RW, RWCRE and RWRE. Marked in boldface are what we consider challenging open problems.

Let us comments on the most relevant items in Table 1.

- Recurrence vs Transience: While for a homogeneous RW we know that it is recurrent if and only if the corresponding local drift is zero, for RWRE the recurrence criterion is encoded in the condition $\langle\log \rho\rangle=0$ (recall Proposition 1.3). In particular, it can happen that the local drift is non-zero, but still the above condition holds and the random walk is recurrent. In fact, a random walk in a non-homogeneous environment builds up non-negligible correlations over time, and its long-time behaviour is a truly global feature. For RWCRE we expect some subtle surprises related to the fluctuations of the corresponding RWRE. In particular, we expect a non-local criterion as for RWRE, controlled by a delicate interplay between the environment law $\mu$ and the cooling map $\tau$. We will address this problem in future work.

- Asymptotic Speed: As for the recurrence criterion, the asymptotic speed of a homogeneous RW is given by its local drift, while for RWRE it is influenced by the presence of the traps. Theorem 1.10 shows that for any cooling map subject to (1.17) RWCRE has the same speed as RWRE. The proof is somewhat delicate and the result itself is surprising, because it means that the speed emerges as a non-local feature (as in the static case), regardless of how fast the resampling increments diverge. In contrast, in the same setup the fluctuations do depend on the cooling map. Furthermore, it is worth noting that more general cooling maps than the ones captured in (1.16)-(1.17) can be considered, e.g. with $\liminf _{k \rightarrow \infty} T_{k}<$ $\infty=\limsup _{k \rightarrow \infty} T_{k}$. In such generality the resulting speed is rather more delicate to analyse (as well as other observables), and can be different from the static speed although still being non-local. For this reason, in this paper we only consider the regularity assumptions in (1.16)-(1.17).

- Large Deviations: Concerning large deviations of order $n$, for homogeneous RW displacements Cramér's theorem tells us that their probabilities decay exponentially fast and are determined by a smooth rate function (see e.g. [9, Chapter I]). On the other hand, as we saw in Section 1.1, large deviations for RWRE are drastically different, both under the quenched and the annealed measure. In particular, both rate functions are non-analytic when $\langle\log \rho\rangle \neq 0$, and contain an interval of zeros when $v_{\mu}>0$. As previously discussed, Theorem 1.11 says that under the quenched law RWCRE satisfies the LDP at rate $n$ with the same rate function. Still, we expect differences between quenched large deviations for RWRE and RWCRE when zooming in on the flat piece, i.e., when considering moderate deviations that decay at a rate slower than $n$. This constitutes yet another interesting open problem. Let us further note that we have not looked at the annealed LDP for RWCRE. We 
RWCRE: ergodic limits and concentration inequalities

expect no surprises, namely, we believe that the annealed rate function for RWCRE is the same as the one for RWRE in Proposition 1.6. In fact, the proof presented in Section 4.2 could be easily adapted (and even significantly simplified) if we had existence and convexity in the annealed setting. In the quenched setting, existence and convexity will be derived by means of the cooling ergodic theorem and standard LDP arguments.

- Fluctuations: We conclude with what we consider to be the most challenging open problem, namely, to characterise the fluctuations for RWCRE, both under the quenched and the annealed measure. Some noteworthy results in this direction were derived in [3], where an annealed CLT for the no-cooling regime was shown [3, Theorem 1.4] and, for $\langle\log \rho\rangle=0$ and $\tau$ growing either polynomially or exponentially, the annealed centered RWCRE displacement was shown to converge to a Gaussian law after an appropriate scaling that depends on $\tau$ [3, Theorem 1.6]. We expect that for sufficiently fast cooling a delicate crossover occurs when $\langle\log \rho\rangle=0$, namely, we expect to see the Kesten [10] limit law as in the static case, at least along certain subsequences. What happens when $\langle\log \rho\rangle \neq 0$ seems to be even more intricate and remains unexplored. In this case for RWRE, Kesten, Kozlov and Spitzer [11] proved that annealed fluctuations can be Gaussian or can be characterised by proper stable law distributions. We expect a rich pallet of behaviour depending on the interplay between $\tau$ and the static limit law. The quenched fluctuations seem even more difficult to analyse in view of the corresponding more delicate results for RWRE (see Zeitouni [16], Ahn and Peterson [1]). As mentioned above, the fact that the fluctuations are affected by the cooling map while the rate function in the LDP is not, is possible because the rate function is non-analytic in the neighbourhood of the speed.

\section{Cooling ergodic theorem}

In this section we prove Theorem 1.12.

We represent the sum in (1.30) as the convex combination

$$
\frac{1}{n}\left(\sum_{k=1}^{\ell(n)-1} \psi_{T_{k}}^{(k)}+\psi_{\bar{T}^{n}}^{(\ell(n))}\right)=\sum_{k=1}^{\ell(n)-1} \frac{T_{k}}{n} \frac{\psi_{T_{k}}^{(k)}}{T_{k}}+\frac{\bar{T}^{n}}{n} \frac{\psi_{\bar{T}^{n}}^{(\ell(n))}}{\bar{T}^{n}},
$$

and use the abbreviations

$$
\gamma_{k, n}=\frac{T_{k}}{n} \mathbb{1}_{\{k \leq \ell(n)-1\}}, \quad \bar{\gamma}^{n}=\frac{\bar{T}^{n}}{n} .
$$

To prove (1.30), we subtract $L$ from (2.1) and center each term in (2.1):

$$
\begin{aligned}
& \sum_{k \in \mathbb{N}} \gamma_{k, n} \frac{\psi_{T_{k}}^{(k)}}{T_{k}}+\bar{\gamma}^{n} \frac{\psi_{\bar{T}^{n}}^{(\ell(n))}}{\bar{T}^{n}}-L \\
& =\underbrace{\sum_{k \in \mathbb{N}} \gamma_{k, n} \mathcal{C}_{k}}_{R_{n}}+\underbrace{\bar{\gamma}^{n} \overline{\mathcal{C}}^{n}}_{B_{n}}+\underbrace{\sum_{k \in \mathbb{N}} \gamma_{k, n}\left(L_{T_{k}}^{(k)}-L\right)+\bar{\gamma}^{n}\left(\bar{L}^{n}-L\right)}_{D_{n}} .
\end{aligned}
$$

Here,

$$
\mathcal{C}_{k}=\mathcal{C}_{T_{k}}^{(k)}, \quad \overline{\mathcal{C}}^{n}=\mathcal{C}_{\bar{T}^{n}}^{(\ell(n))}, \quad \bar{L}^{n}=L_{\bar{T}^{n}}^{(\ell(n))},
$$

with

$$
\mathcal{C}_{T}^{(k)}=\frac{\psi_{T}^{(k)}}{T}-L_{T}^{(k)}, \quad L_{T}^{(k)}=\mathbb{E}\left[\frac{\psi_{T}^{(k)}}{T}\right], \quad T \in \mathbb{N} .
$$


The terms $R_{n}, B_{n}$ and $D_{n}$ correspond to refreshed, boundary and deterministic increments, respectively. In Sections 2.1, 2.2 and 2.3 we treat each of these terms separately, and show that they are asymptotically vanishing.

\subsection{Refreshed term}

We next show that

$$
\limsup _{n \rightarrow \infty}\left|R_{n}\right|=0 \quad \mathbb{P}-\text { a.s. }
$$

In view of (1.29), we split the increments of the resampling times according to a growth parameter $\gamma>0$ such that $\gamma \delta>1$,

$$
\sum_{k \in \mathbb{N}} \gamma_{k, n} \mathcal{C}_{k}=\underbrace{\sum_{k \in \mathbb{N}} \gamma_{k, n} \mathcal{C}_{k} \mathbb{1}_{\left\{T_{k} \geq k^{\gamma}\right\}}}_{R_{n}^{L}}+\underbrace{\sum_{k \in \mathbb{N}} \gamma_{k, n} \mathcal{C}_{k} \mathbb{1}_{\left\{T_{k}<k^{\gamma}\right\}}}_{R_{n}^{S}}
$$

which corresponds to the sum of large and small increments, respectively. The goal is to bound both $\lim \sup _{n \rightarrow \infty}\left|R_{n}^{L}\right|$ and $\limsup _{n \rightarrow \infty}\left|R_{n}^{S}\right|$.

We first treat the sum of large increments $R_{n}^{L}$. Note that Assumption (A2) and (1.29) imply that $L_{T}^{(k)} \rightarrow L$ as $T \rightarrow \infty$ uniformly in $k \in \mathbb{N}$. Therefore, using the triangle inequality, (1.29) can be written as

$$
\sup _{k \in \mathbb{N}} \mathbb{P}\left(\left|C_{T}^{(k)}\right|>\varepsilon\right)<\frac{C^{\prime}}{T^{\delta}}
$$

Hence, if $\gamma \delta>1$, then

$$
\sum_{k \in \mathbb{N}} \mathbb{P}\left(\left|\mathcal{C}_{k}\right| \mathbb{1}_{\left\{T_{k} \geq k^{\gamma}\right\}}>\varepsilon\right)<\sum_{k \in \mathbb{N}} \frac{C^{\prime}}{k^{\gamma \delta}}<\infty
$$

Applying the Borel-Cantelli lemma, we get

$$
\limsup _{k \rightarrow \infty} \mathcal{C}_{k} \mathbb{1}_{\left\{T_{k} \geq k^{\gamma}\right\}} \leq \varepsilon \quad \mathbb{P}-\text { a.s. }
$$

Since $\lim _{n \rightarrow \infty} \gamma_{k, n}=0$ for fixed $k \in \mathbb{N}$ and $\sum_{k \in \mathbb{N}} \gamma_{k, n} \leq 1$, we obtain

$$
\limsup _{n \rightarrow \infty}\left|R_{n}^{L}\right| \leq \varepsilon \quad \mathbb{P}-\text { a.s. }
$$

To deal with the sum of small increments $R_{n}^{S}$, we apply the Markov inequality:

$$
\mathbb{P}\left(\left|R_{n}^{S}\right|>\varepsilon\right) \leq \frac{1}{\varepsilon^{2 N}} \mathbb{E}\left[\left(\sum_{k=1}^{n} \gamma_{k, n}^{S} \mathcal{C}_{k}\right)^{2 N}\right], \quad \gamma_{k, n}^{S}=\gamma_{k, n} \mathbb{1}_{\left\{T_{k}<k^{\gamma}\right\}}
$$

Since the $\mathcal{C}_{k}$ 's are independent, zero-mean and bounded random variables, when we expand the $2 N$-th power, all terms with first moment disappear. Therefore, we can 
RWCRE: ergodic limits and concentration inequalities

estimate the moments as ( $C$ is a generic constant that may change from line to line)

$$
\begin{aligned}
& \mathbb{E}\left[\left(\sum_{k=1}^{n} \gamma_{k, n}^{S} \mathcal{C}_{k}\right)^{2 N}\right] \\
& =\sum_{m=1}^{N} \sum_{\substack{\ell_{1}, \cdots, \ell_{m} \in \mathbb{N} \backslash\{1\} \\
\ell_{1}+\cdots+\ell_{m}=2 N}}\left(\begin{array}{c}
2 N \\
\ell_{1} \cdots \ell_{m}
\end{array}\right) \sum_{n \geq k_{1}>\ldots>k_{m}} \mathbb{E}\left[\left(\gamma_{k_{1}, n}^{S} \mathcal{C}_{k_{1}}\right)^{\ell_{1}} \cdots\left(\gamma_{k_{m}, n}^{S} \mathcal{C}_{k_{m}}\right)^{\ell_{m}}\right] \\
& \leq \sum_{m=1}^{N} \sum_{\substack{\ell_{1}, \cdots, \ell_{m} \in \mathbb{N} \backslash\{1\} \\
\ell_{1}+\cdots+\ell_{m}=2 N}}\left(\begin{array}{c}
2 N \\
\ell_{1} \cdots \ell_{m}
\end{array}\right) \sum_{n \geq k_{1}>\cdots>k_{m}}\left(C n^{\gamma-1}\right)^{2 N} \\
& \leq\left(C n^{\gamma-1}\right)^{2 N} \sum_{m=1}^{N} n_{\substack{m \\
\ell_{1}, \cdots, \ell_{m} \in \mathbb{N} \backslash\{1\} \\
\ell_{1}+\cdots+\ell_{m}=2 N}}\left(\begin{array}{c}
2 N \\
\ell_{1} \cdots \ell_{m}
\end{array}\right) \leq c_{N} n^{N(2 \gamma-1)},
\end{aligned}
$$

where in the first inequality we use the bounds $\mathcal{C}_{k_{i}} \leq C$ (from Assumption (A2)) and $\gamma_{k_{i}, n}^{S} \leq \frac{k_{i}^{\gamma}}{n}$ for $i=1, \ldots, m$, in the second inequality the bound

$$
\sum_{n \geq k_{1}>\ldots>k_{m}} 1 \leq n^{m},
$$

and in the third inequality the bound $m \leq N$ and the abbreviation

$$
c_{N}=C^{2 N} \sum_{m=1}^{N} \sum_{\substack{m, \cdots, \ell_{m} \in \mathbb{N} \backslash\{1\} \\
\ell_{1}+\cdots+\ell_{m}=2 N}}\left(\begin{array}{c}
2 N \\
\ell_{1} \cdots \ell_{m}
\end{array}\right) .
$$

The right-hand side of (2.13) is summable in $n$ as long as $\gamma<\frac{1}{2}$, because we can choose $N$ arbitrarily large. This suggests that we need to further separate the argument.

Case $\delta>2$ : single split. If $\delta>2$, then we can pick $\gamma<\frac{1}{2}$. From (2.12) and (2.13), we obtain

$$
\sum_{n \in \mathbb{N}} \mathbb{P}\left(\left|R_{n}^{S}\right|>\varepsilon\right)<\infty .
$$

Hence, by the Borel-Cantelli lemma,

$$
\limsup _{n \rightarrow \infty}\left|R_{n}^{S}\right| \leq \varepsilon \quad \mathbb{P}-\text { a.s. }
$$

Combining (2.7), (2.11) and (2.17), we get that for $\delta>2$,

$$
\limsup _{n \rightarrow \infty}\left|R_{n}\right| \leq 2 \varepsilon \quad \mathbb{P}-\text { a.s. }
$$

Case $\delta<2$ : multi-layer split. If $\delta<2$, then we must pick $\gamma>\frac{1}{2}$ to satisfy $\gamma \delta>1$. Here we can no longer use the previous argument to obtain (2.16). To overcome this difficulty, we implement a multi-layer scheme distinguishing small and large increments according to a growth parameter. Take $M \in \mathbb{N}$ such that $\frac{M}{3} \delta>1$. Similarly to (2.7), define the first split:

$$
R_{n}=\sum_{k \in \mathbb{N}} \gamma_{k, n} \mathcal{C}_{k}=\underbrace{\sum_{k \in \mathbb{N}} \gamma_{k, n}^{1, L} \mathcal{C}_{k}}_{R_{n}^{1, L}}+\underbrace{\sum_{k \in \mathbb{N}} \gamma_{k, n}^{1, S} \mathcal{C}_{k}}_{R_{n}^{1, S}}
$$


RWCRE: ergodic limits and concentration inequalities

where

$$
\gamma_{k, n}^{1, S}=\gamma_{k, n} \mathbb{1}_{\left\{T_{k}<k^{1 / 3}\right\}}, \quad \gamma_{k, n}^{1, L}=\gamma_{k, n} \mathbb{1}_{\left\{T_{k} \geq k^{1 / 3}\right\}} .
$$

1. To estimate $R_{n}^{1, S}$, as in (2.12) and (2.13) we apply the Markov inequality, estimate the moments and obtain

$$
\mathbb{P}\left(\left|R_{n}^{1, S}\right|>\varepsilon\right) \leq c_{N} n^{-N / 3} .
$$

Since we can choose $N>3$ in (2.21), we conclude that $\mathbb{P}\left(\left|R_{n}^{1, S}\right|>\varepsilon\right)$ is summable in $n$ and therefore, by the Borel-Cantelli lemma,

$$
\limsup _{n \rightarrow \infty}\left|R_{n}^{1, S}\right| \leq \varepsilon \quad \mathbb{P}-\text { a.s. }
$$

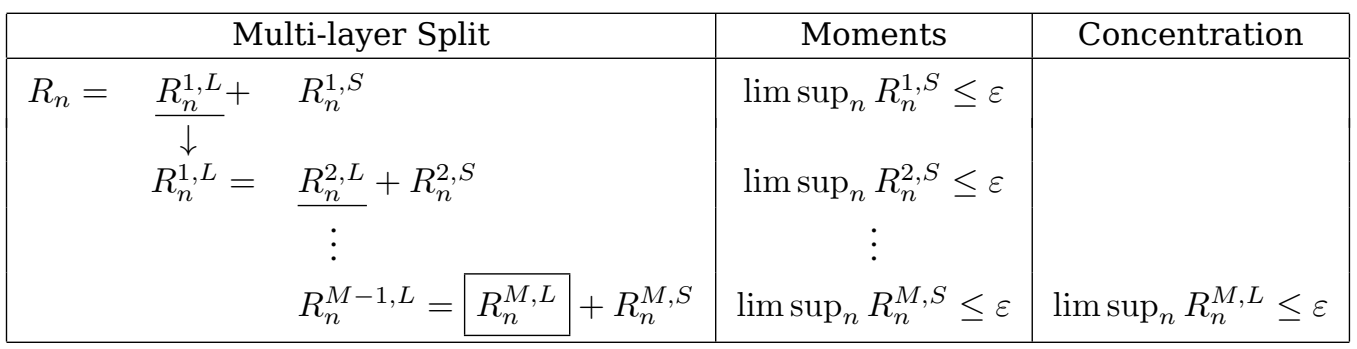

Table 2: Splitting scheme.

2. To estimate $R_{n}^{1, L}$, the idea is to it decompose iteratively, as we did with $R_{n}$ in (2.19), and control the small increments with moment bounds until we can apply concentration estimates. The resulting scheme is summarised in Table 2.

2a. To build the second split, we relabel the terms in $R_{n}^{1, L}$, i.e., we choose an ordered subsequence $\left(k_{j}^{1}\right)_{j \in \mathbb{N}}$ such that

$$
\left\{k_{1}^{1}, k_{2}^{1}, \ldots\right\}=\left\{j \in \mathbb{N}: T_{j} \geq j^{1 / 3}\right\} .
$$

If this is finite, then $\lim _{n \rightarrow \infty} R_{n}^{1, L}=0$. Thus, we need only consider the case where (2.23) is infinite. Denoting by $J(1 ; n)$ the cardinality of $\left\{k_{j}^{1}: \tau\left(k_{j}^{1}\right) \leq n\right\}$, we define the second split:

where

$$
R_{n}^{1, L}=\sum_{j=1}^{J(1 ; n)} \gamma_{k_{j}^{1}, n} \mathcal{C}_{k_{j}^{1}}=\underbrace{\sum_{j=1}^{J(1 ; n)} \gamma_{k_{j}^{1}, n}^{2, L} \mathcal{C}_{k_{j}^{1}}}_{R_{n}^{2, L}}+\underbrace{\sum_{j=1}^{J(1 ; n)} \gamma_{k_{j}^{1}, n}^{2, S} \mathcal{C}_{k_{j}^{1}}}_{R_{n}^{2, S}},
$$

$$
\gamma_{k_{j}^{1}, n}^{2, S}=\gamma_{k_{j}^{1}, n} \mathbb{1}_{\left\{T_{k_{j}^{1}}<j^{2 / 3}\right\}}, \quad \gamma_{k_{j}^{1}, n}^{2, L}=\gamma_{k_{j}^{1}, n} \mathbb{1}_{\left\{T_{k_{j}^{1}} \geq j^{2 / 3}\right\}} .
$$

Next, we abbreviate $n(1 ; J)=\inf \{n: J(1 ; n)=J\}$. Then, since

$$
\limsup _{n \rightarrow \infty}\left|R_{n}^{2, S}\right|=\limsup _{J \rightarrow \infty}\left|R_{n(1 ; J)}^{2, S}\right|,
$$

it suffices to show that $\limsup _{J \rightarrow \infty}\left|R_{n(1 ; J)}^{2, S}\right| \leq \varepsilon \mathbb{P}-$ a.s. Note that, since $T_{k_{j}^{1}} \geq j^{1 / 3}$, we have a lower bound on $n(1 ; J)$ :

$$
n(1 ; J) \geq \sum_{j=1}^{J} T_{k_{j}^{1}} \geq \sum_{j=1}^{J} j^{1 / 3} \geq c J^{4 / 3},
$$

which yields

$$
\gamma_{k_{j}^{1}, n(1 ; J)}^{2, S} \leq \frac{j^{2 / 3}}{c J^{4 / 3}} \leq \frac{1}{c J^{2 / 3}}
$$


RWCRE: ergodic limits and concentration inequalities

Similarly to the first split, we apply the Markov inequality

$$
\mathbb{P}\left(\left|R_{n(1 ; J)}^{2, S}\right|>\varepsilon\right) \leq \frac{1}{\varepsilon^{2 N}} \mathbb{E}\left[\left(\sum_{j=1}^{J} \gamma_{k_{j}^{1}, n}^{2, S} \mathcal{C}_{k_{j}^{1}}\right)^{2 N}\right]
$$

and estimate moments

$$
\mathbb{E}\left[\left(\sum_{j=1}^{J} \gamma_{k_{j}^{1}, n(1 ; J)}^{2, S} \mathcal{C}_{k_{j}^{1}}\right)^{2 N}\right] \leq c_{N} J^{-N / 3} .
$$

Once we choose $N>3$, this becomes summable in $J$. Therefore, by the Borel-Cantelli lemma and (2.26), we obtain

$$
\limsup _{n \rightarrow \infty}\left|R_{n}^{2, S}\right| \leq \varepsilon \quad \mathbb{P}-\text { a.s. }
$$

2b. We continue the induction step. For any $i<M$, after bounding $\lim \sup _{n \rightarrow \infty}\left|R_{n}^{i, S}\right|$, we relabel the terms in $R_{n}^{i, L}$ and define

$$
\left\{k_{1}^{i}, k_{2}^{i}, \ldots\right\}=\left\{j \in \mathbb{N}: T_{k_{j}^{i-1}} \geq j^{i / 3}\right\} .
$$

If this is finite, then $\lim _{n \rightarrow \infty} R_{n}^{i-1, L}=0$. Denoting by $J(i ; n)$ the cardinality of $\left\{k_{j}^{i}: \tau\left(k_{j}^{i}\right) \leq\right.$ $n\}$, we define the $(i+1)$-st split:

$$
R_{n}^{i, L}=\sum_{j=1}^{J(i ; n)} \gamma_{k_{j}^{i}, n} \mathcal{C}_{k_{j}^{i}}=\underbrace{\sum_{j=1}^{J(i ; n)} \gamma_{k_{j}^{i}, n}^{i+1, L} \mathcal{C}_{k_{j}^{i}}}_{R_{n}^{i+1, L}}+\underbrace{\sum_{j=1}^{J(i ; n)} \gamma_{k_{j}^{i}, n}^{i+1, S} \mathcal{C}_{k_{j}^{i}}}_{R_{n}^{i+1, S}}
$$

where

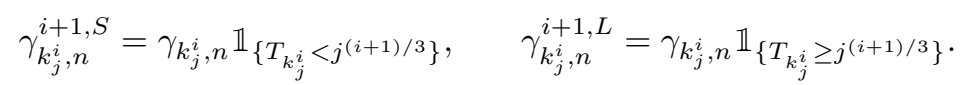

Let $n(i ; J)=\inf \{n: J(i ; n)=J\}$. Then, by a similar computation as in (2.27) and (2.28), we have the following bounds:

$$
n(i ; J) \geq c J^{1+i / 3}, \quad \gamma_{k_{j}^{i}, n(i ; J)}^{i+1, S} \leq \frac{1}{c J^{2 / 3}} .
$$

Using the Markov inequality and moments bounds, we obtain

$$
\sum_{J \in \mathbb{N}} \mathbb{P}\left(\left|R_{n(i ; J)}^{i+1, S}\right|>\varepsilon\right)<\infty
$$

Therefore we conclude that

$$
\limsup _{n \rightarrow \infty}\left|R_{n}^{i+1, S}\right| \leq \varepsilon \quad \mathbb{P}-\text { a.s. }
$$

2c. Once we bound $\lim \sup _{n \rightarrow \infty}\left|R_{n}^{M, S}\right|$, we are left with the term $R_{n}^{M, L}$. Since

$$
R_{n}^{M, L}=\sum_{j \in \mathbb{N}} \gamma_{k_{j}^{M}, n} \mathbb{1}_{\left\{T_{k_{j}^{M-1}>j^{M / 3}}\right\}} \mathcal{C}_{k_{j}^{M-1}}
$$

and $\frac{M}{3} \delta>1$, we apply (1.29) to obtain

$$
\sum_{j \in \mathbb{N}} \mathbb{P}\left(\left|\mathcal{C}_{k_{j}^{M-1}}\right| \mathbb{1}_{\left\{T_{k_{j}^{M-1}>j}^{M / 3}\right\}}>\varepsilon\right)<\infty .
$$


RWCRE: ergodic limits and concentration inequalities

Hence, by the Borel-Cantelli lemma,

$$
\limsup _{j \rightarrow \infty}\left|\mathcal{C}_{k_{j}^{M-1}}\right| \mathbb{1}_{\left\{T_{k_{j}^{M-1}>j^{M / 3}}\right\}} \leq \varepsilon \quad \mathbb{P}-\text { a.s. }
$$

Since $\lim _{n \rightarrow \infty} \gamma_{k, n}=0$ for fixed $k$ and $\sum_{k \in \mathbb{N}} \gamma_{k, n} \leq 1$, we obtain

$$
\limsup _{n \rightarrow \infty}\left|R_{n}^{M, L}\right| \leq \varepsilon \quad \mathbb{P}-\text { a.s. }
$$

3. Combining (2.41) and (2.37) for $i<M$, we conclude that

$$
\limsup _{n \rightarrow \infty}\left|R_{n}\right| \leq(M+1) \varepsilon \quad \mathbb{P} \text { - a.s. }
$$

and (2.6) follows since $\varepsilon>0$ is arbitrary.

\subsection{Boundary term}

We next show that

$$
\limsup _{n \rightarrow \infty}\left|B_{n}\right|=0 \quad \mathbb{P}-\text { a.s. }
$$

Let $V_{k}=\sup \left\{\bar{\gamma}^{n}\left|\overline{\mathcal{C}}^{n}\right|: n \in I_{k}\right\}$. Because $\cup_{k \in \mathbb{N}} I_{k}=\mathbb{R}_{+}$(see (1.16)), we have

$$
\limsup _{n \rightarrow \infty} \bar{\gamma}^{n}\left|\overline{\mathcal{C}}^{n}\right|=\limsup _{k \rightarrow \infty} V_{k}
$$

It therefore suffices to show that for arbitrary $\varepsilon>0$,

$$
\limsup _{k \rightarrow \infty} V_{k} \leq \varepsilon \quad \mathbb{P}-\text { a.s. }
$$

If $\bar{\gamma}^{n} \leq \varepsilon$, then using Assumption (A2) we can bound $\left|B_{n}\right| \leq C \varepsilon$. Therefore, for $n \in I_{k}$ we only need to consider $\bar{\gamma}^{n}>\varepsilon$ (see Fig. 5), in which case we see that

$$
n>\frac{\tau(k-1)}{1-\varepsilon}=N_{k, \varepsilon} .
$$
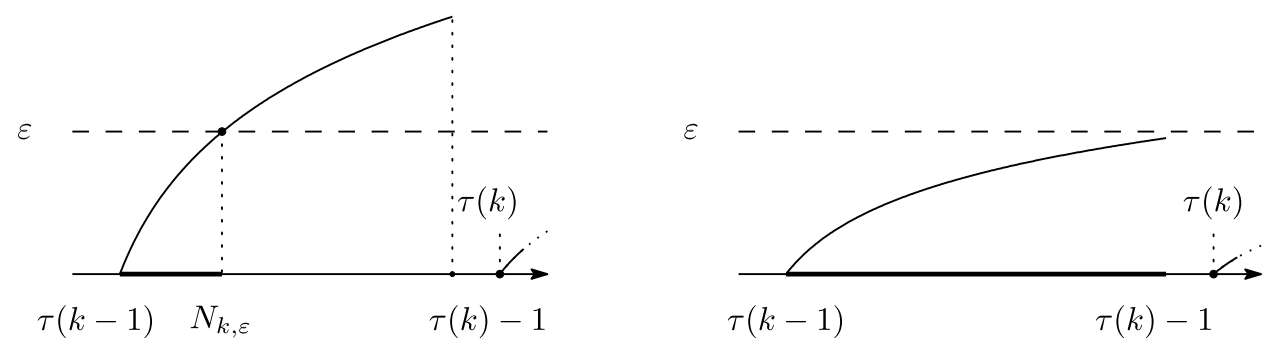

Figure 5: The curve of $\bar{\gamma}^{n}$. Left: If $N_{k, \varepsilon}<\tau(k)$, then $\bar{\gamma}_{n} \leq \varepsilon$ for $n \in\left[\tau(k-1), N_{k, \varepsilon}\right)$ and $\bar{\gamma}_{n}>\varepsilon$ for $n \in\left[N_{k, \varepsilon}, \tau(k)\right)$. Right: If $N_{k, \varepsilon} \geq \tau(k)$, then $\bar{\gamma}_{n} \leq \varepsilon$ for all $n \in I_{k}$.

If $\tau(k) \leq N_{k, \varepsilon}$, then the interval $I_{k}$ can be ignored. Defining

$$
\left\{k_{1}, k_{2}, \ldots\right\}=\left\{k \in \mathbb{N}: \tau(k) \geq N_{k, \varepsilon}\right\},
$$

our task reduces to showing that

$$
\limsup _{j \rightarrow \infty} V_{k_{j}} \leq C \varepsilon \quad \mathbb{P}-\text { a.s. }
$$


RWCRE: ergodic limits and concentration inequalities

Note that the subsequence $\left(\tau\left(k_{j}\right)\right)_{j \in \mathbb{N}}$ grows at least exponentially fast once

$$
\tau\left(k_{j}\right) \geq N_{k_{j}, \varepsilon}>(1+\varepsilon) \tau\left(k_{j}-1\right) \geq(1+\varepsilon) \tau\left(k_{j-1}\right) \geq(1+\varepsilon)^{j-1} \tau\left(k_{1}\right) .
$$

Since $\left|B_{n}\right| \leq C \varepsilon$ for $n \in\left[\tau\left(k_{j}-1\right), N_{k_{j}, \varepsilon}\right)$ and $\bar{\gamma}^{n} \leq 1$, by letting $m=\bar{T}^{n}=n-\tau\left(k_{j}-1\right)$ and noting that $(1+\varepsilon) \tau\left(k_{j}-1\right) \leq N_{k, \varepsilon}$, we obtain

$$
\begin{aligned}
\mathbb{P}\left(V_{k_{j}}>C \varepsilon\right) & =\mathbb{P}\left(\sup _{N_{k, \varepsilon} \leq n<\tau\left(k_{j}\right)} \bar{\gamma}_{n}\left|\bar{C}^{n}\right|>C \varepsilon\right) \leq \mathbb{P}\left(\sup _{N_{k, \varepsilon} \leq n<\tau\left(k_{j}\right)}\left|\bar{C}^{n}\right|>C \varepsilon\right) \\
& \leq \mathbb{P}\left(\sup _{\varepsilon \tau\left(k_{j}-1\right) \leq m<T_{k_{j}}}\left|\frac{\psi_{m}^{\left(k_{j}\right)}}{m}-\mathbb{E}\left[\frac{\psi_{m}^{\left(k_{j}\right)}}{m}\right]\right|>C \varepsilon\right) .
\end{aligned}
$$

By the union bound applied to (2.50), we arrive at

$$
\mathbb{P}\left(V_{k_{j}}>C \varepsilon\right) \leq \sum_{m=\varepsilon \tau\left(k_{j}-1\right)}^{T_{k_{j}}} \mathbb{P}\left(\left|\frac{\psi_{m}^{\left(k_{j}\right)}}{m}-\mathbb{E}\left[\frac{\psi_{m}^{\left(k_{j}\right)}}{m}\right]\right|>C \varepsilon\right) .
$$

By (2.8),

$$
\mathbb{P}\left(V_{k_{j}}>C \varepsilon\right) \leq \sum_{m=\varepsilon \tau\left(k_{j}-1\right)}^{T_{k_{j}}} \frac{\widetilde{C}}{m^{\delta}},
$$

for some $\widetilde{C}=C^{\prime}(C \varepsilon)>0$ not depending on $k_{j}$.

Case $\delta>1$. From (2.52) we see that

$$
\mathbb{P}\left(V_{k_{j}}>C \varepsilon\right) \leq \frac{C^{\prime \prime}}{\varepsilon \tau\left(k_{j}-1\right)^{\delta-1}} .
$$

If $\delta>1$, then together with (2.49) this implies that (2.53) is summable in $j$. Hence, by the Borel-Cantelli lemma, we obtain

$$
\limsup _{j \rightarrow \infty} V_{k_{j}} \leq C \varepsilon \quad \mathbb{P} \text { - a.s. }
$$

Case $\delta<1$. In this case, we need a more refined argument to prove (2.43). To control the boundary term on the interval $I_{k_{j}}$, we construct a sequence of times $\left(J_{i}\right)_{i \in \mathbb{N}_{0}}$ such that $J_{0}=\varepsilon \tau\left(k_{j}-1\right)$ and $J_{i}=(1+\varepsilon) J_{i-1}$. For $m \in\left(J_{i}, J_{i+1}\right)$, using (1.31) and the triangle inequality, we obtain that

$$
\left|\frac{\psi_{m}^{\left(k_{j}\right)}}{m}-\frac{\psi_{J_{i}}^{\left(k_{j}\right)}}{J_{i}}\right| \leq 2 C \varepsilon
$$

Hence

$$
\begin{aligned}
\left|\frac{\psi_{m}^{\left(k_{j}\right)}}{m}-\mathbb{E}\left[\frac{\psi_{m}^{\left(k_{j}\right)}}{m}\right]\right| & \leq\left|\frac{\psi_{m}^{\left(k_{j}\right)}}{m}-\frac{\psi_{J_{i}}^{\left(k_{j}\right)}}{J_{i}}\right|+\left|\frac{\psi_{J_{i}}^{\left(k_{j}\right)}}{J_{i}}-\mathbb{E}\left[\frac{\psi_{J_{i}}^{\left(k_{j}\right)}}{J_{i}}\right]\right|+\left|\mathbb{E}\left[\frac{\psi_{m}^{\left(k_{j}\right)}}{m}-\frac{\psi_{J_{i}}^{\left(k_{j}\right)}}{J_{i}}\right]\right| \\
& \leq\left|\frac{\psi_{J_{i}}^{\left(k_{j}\right)}}{J_{i}}-\mathbb{E}\left[\frac{\psi_{J_{i}}^{\left(k_{j}\right)}}{J_{i}}\right]\right|+4 C \varepsilon .
\end{aligned}
$$

Therefore

$$
\left\{\sup _{\varepsilon \tau\left(k_{j}-1\right) \leq m<T_{k_{j}}}\left|\frac{\psi_{m}^{\left(k_{j}\right)}}{m}-\mathbb{E}\left[\frac{\psi_{m}^{\left(k_{j}\right)}}{m}\right]\right|>5 C \varepsilon\right\} \subset\left\{\sup _{i \in \mathbb{N}_{0}}\left|\frac{\psi_{J_{i}}^{\left(k_{j}\right)}}{J_{i}}-\mathbb{E}\left[\frac{\psi_{J_{i}}^{\left(k_{j}\right)}}{J_{i}}\right]\right|>C \varepsilon\right\} .
$$


Hence, arguing as in (2.50) and using (2.57), the union bound and (2.8), we can estimate

$$
\begin{aligned}
\mathbb{P}\left(V_{k_{j}}>5 C \varepsilon\right) & =\mathbb{P}\left(\sup _{\varepsilon \tau\left(k_{j}-1\right) \leq m<T_{k_{j}}}\left|\frac{\psi_{m}^{\left(k_{j}\right)}}{m}-\mathbb{E}\left[\frac{\psi_{m}^{\left(k_{j}\right)}}{m}\right]\right|>5 C \varepsilon\right) \\
& \leq \mathbb{P}\left(\exists i \in \mathbb{N}_{0}:\left|\frac{\psi_{J_{i}}^{\left(k_{j}\right)}}{J_{i}}-\mathbb{E}\left[\frac{\psi_{J_{i}}^{\left(k_{j}\right)}}{J_{i}}\right]\right|>C \varepsilon\right) \\
& \leq \sum_{i \in \mathbb{N}_{0}} \frac{C^{\prime}}{J_{i}^{\delta}}=\sum_{i \in \mathbb{N}_{0}} \frac{C^{\prime}}{(1+\varepsilon)^{i \delta}\left(\varepsilon \tau\left(k_{j}-1\right)\right)^{\delta}}
\end{aligned}
$$

which is summable in $j$ due to (2.49). By the Borel-Cantelli lemma, we conclude that

$$
\limsup _{n \rightarrow \infty}\left|B_{n}\right| \leq 5 C \varepsilon \quad \mathbb{P}-\text { a.s. }
$$

Since $\varepsilon>0$ is arbitrary, (2.59) and (2.54) imply (2.43).

\subsection{Deterministic term}

To conclude the proof of Theorem 1.12, it remains to show that

$$
\lim _{n \rightarrow \infty}\left(\sum_{k \in \mathbb{N}} \gamma_{k, n} L_{T_{k}}^{(k)}+\bar{\gamma}^{n} \bar{L}^{n}\right)=L
$$

where $\gamma_{k, n}, \bar{\gamma}^{n}$ is defined in (2.2), $\bar{T}^{n}$ in (1.22), $L_{T_{k}}^{(k)}$ in (2.5) and $\bar{L}^{n}$ in (2.4). First we recall that (1.29) and (A2) imply that $L_{T_{k}}^{(k)}-L \rightarrow 0$ uniformly in $k$. Since $\gamma_{k, n} \rightarrow 0$ as $n \rightarrow \infty$ and $\sum_{k \in \mathbb{N}} \gamma_{n} \leq 1$, by the Toeplitz Lemma[13, Thm.1.2.3, p. 36], we obtain

$$
\lim _{n \rightarrow \infty} \sum_{k \in \mathbb{N}} \gamma_{k, n}\left(L_{T_{k}}^{(k)}-L\right)=0
$$

As for the boundary part, if $\bar{\gamma}^{n}>\varepsilon$, then there is an $N \in \mathbb{N}$ such that for any $n>N$, $\left|\bar{L}^{n}-L\right| \leq \varepsilon$. Otherwise, $\bar{\gamma}^{n}\left|\bar{L}^{n}-L\right| \leq 2 \varepsilon$. Since $\varepsilon>0$ is arbitrary, we conclude (2.60).

Combining (2.6), (2.43) and (2.61), we get the claim in (1.30).

\section{Concentration for RWRE}

In this section we prove Theorem 1.13. We start in Section 3.1 by proving (1.32). In fact, as is usual in the context of RWRE, hitting times are easier to handle and their concentration will follow from an adaptation of an argument presented in the proof of Zeitouni [Lemma 3.4.10][16]. In Sections 3.3 and 3.4 we will show (1.33) and (1.34), respectively. Their proofs will be based on a key lemma, Lemma 3.1, which we state below.

Lemma 3.1 (Concentration of empirical speed on an interval).

Suppose that $\mu$ is $\alpha$-basic. Let $\delta>0$, and $\Delta=(a, b]$ or $\Delta=[a, b]$ with $a \neq b$.

- If $0 \leq a$, then for every $\varepsilon>0$ there are positive constants $C, c$ such that

$$
\mu\left(\omega:\left|\frac{1}{n} \log P_{0}^{\omega}\left(\frac{Z_{n}}{n} \in \Delta\right)+\mathcal{I}(a)\right|>\varepsilon\right) \leq C e^{-c n^{1-\delta}}, \quad \forall n \in \mathbb{N} .
$$

- If $b \leq 0$, then for every $\varepsilon>0$ there are positive constants $C, c$ such that

$$
\mu\left(\omega:\left|\frac{1}{n} \log P_{0}^{\omega}\left(\frac{Z_{n}}{n} \in \Delta\right)+\mathcal{I}(b)\right|>\varepsilon\right) \leq C e^{-c n^{1-\delta}}, \quad \forall n \in \mathbb{N} .
$$


RWCRE: ergodic limits and concentration inequalities

\subsection{Concentration for hitting times}

Next we prove (1.32). The following argument is similar to [16, Lemma 3.4.10, p. 291].

Define, for fixed $K \in(0, \infty)$,

$$
g^{\delta, n}(\omega)=\log E_{0}^{\omega}\left[e^{\lambda H_{n}} \mathbb{1}_{\left\{H_{n}<K n\right\}} \mathbb{1}_{\left\{N^{n}<n^{\delta / 2}\right\}}\right],
$$

where $N^{n}=\sup _{x \in \mathbb{Z}} N_{x}^{n}$ and $N_{x}^{n}$ is the number of visits at $x$ before $H_{n}$. Note that $g^{\delta, n}(\omega)$ is a function of the environment coordinates $\left(\omega_{i}:|i| \leq K n\right)$. For $i \in \mathbb{N}$, define

$$
\begin{aligned}
& \mathcal{F}_{0}=\sigma\{\emptyset\}, \quad \mathcal{F}_{1}=\sigma\left\{\omega_{0}\right\}, \quad \mathcal{F}_{2}=\sigma\left\{\omega_{0}, \omega_{1}\right\}, \quad \mathcal{F}_{3}=\sigma\left\{\omega_{0}, \omega_{1}, \omega_{-1}\right\}, \\
& \quad \vdots \\
& \mathcal{F}_{i}=\sigma\left\{\omega_{j}: j \in(-\lceil i / 2\rceil,\lfloor i / 2\rfloor] \cap \mathbb{Z}\right\},
\end{aligned}
$$

and denote by $E^{\mu}$ expectation with respect to $\mu$. Then

$$
E^{\mu}\left[g^{\delta, n} \mid \mathcal{F}_{2 K n}\right]=g^{\delta, n}, \quad E^{\mu}\left[g^{\delta, n} \mid \mathcal{F}_{0}\right]=E^{\mu}\left[g^{\delta, n}\right] .
$$

Rewrite

$$
g^{\delta, n}(\omega)-E^{\mu}\left[g^{\delta, n}\right]=\sum_{i=1}^{2 K n} d_{i}(\omega)
$$

with

$$
d_{i}(\omega)=E^{\mu}\left[g^{\delta, n} \mid \mathcal{F}_{i}\right](\omega)-E^{\mu}\left[g^{\delta, n} \mid \mathcal{F}_{i-1}\right](\omega) .
$$

Let $S_{0}=E^{\mu}\left[g^{\delta, n}\right]$ and $S_{m}=\sum_{i=1}^{m} d_{i}(\omega)$. Since $E^{\mu}\left[d_{i} \mid \mathcal{F}_{m}\right]=0$ for $i>m,\left\{S_{m}\right\}_{m \in \mathbb{N}_{0}}$ is a martingale. We obtain a bound for $d_{i}(\omega)$ by writing

$$
d_{i+1}(\omega)=E^{\mu}\left[g^{\delta, n} \mid \mathcal{F}_{i+1}\right](\omega)-E^{\mu}\left[g^{\delta, n} \mid \mathcal{F}_{i}\right](\omega) \leq \sup _{\omega^{i}}\left[g^{\delta, n}\left(\omega^{i}\right)-g^{\delta, n}(\omega)\right]=:\left|d_{i}\right|_{\infty},
$$

where $\omega_{x}^{i}=\omega_{x}$ for all $x \in \mathbb{Z}$, except for

$$
x_{i}= \begin{cases}-i / 2, & \text { if } i \text { is even, } \\ \lceil i / 2\rceil, & \text { if } i \text { is odd. }\end{cases}
$$

To compute the difference in (3.8), we use a bound on the derivative of $g^{\delta, n}(\omega)$. From the computations in [16, p. 291] we have that, for any $\delta \in(0,1)$,

$$
\left|d_{i}\right|_{\infty} \leq\left|\frac{\partial g^{\delta, n}(\omega)}{\partial \omega_{x_{i}}}\right| \leq \sqrt{K} \frac{n^{\delta / 2}}{\mathfrak{c}} .
$$

Applying the Azuma-Hoeffding inequality, we obtain

$$
\mu\left(\omega:\left|S_{2 K n}-S_{0}\right|>u\right) \leq 2 \exp \left(-\frac{u^{2}}{2 \sum_{i=1}^{2 K n}\left|d_{i}\right|_{\infty}^{2}}\right) .
$$

Since $S_{2 K n}=g^{\delta, n}(\omega)$ and $S_{0}=E^{\mu}\left[g^{\delta, n}\right]$, we obtain

$$
\mu\left(\omega:\left|g^{\delta, n}(\omega)-E^{\mu}\left[g^{\delta, n}\right]\right|>u n\right) \leq 2 \exp \left(-\frac{u^{2} n^{2}}{C n^{1+\delta}}\right) \leq 2 \exp \left(-\frac{u^{2}}{C} n^{1-\delta}\right) .
$$

To conclude the proof of (1.32), we write

$$
\begin{aligned}
& \mu\left(\omega:\left|\frac{1}{n} \log E_{0}^{\omega}\left[e^{\lambda H_{n}}\right]-\mathcal{J}^{*}(\lambda)\right|>\varepsilon\right) \leq \mu\left(\omega: \frac{1}{n}\left|\log E_{0}^{\omega}\left[e^{\lambda H_{n}}\right]-g^{\delta, n}(\omega)\right|>\frac{1}{3} \varepsilon\right) \\
& +\mu\left(\omega: \frac{1}{n}\left|g^{\delta, n}(\omega)-E^{\mu}\left[g^{\delta, n}\right]\right|>\frac{1}{3} \varepsilon\right)+\mu\left(\omega:\left|\frac{1}{n} E^{\mu}\left[g^{\delta, n}\right]-\mathcal{J}^{*}(\lambda)\right|>\frac{1}{3} \varepsilon\right) .
\end{aligned}
$$


We will estimate the second term in the right hand side by (3.12). Let us first show that the first and the third term vanish as $n \rightarrow \infty$. For the first term in (3.13), the ellipticity condition implies that for large $n$,

$$
\frac{1}{n}\left|\log E_{0}^{\omega}\left[e^{\lambda H_{n}}\right]-g^{\delta, n}(\omega)\right|<\frac{1}{3} \varepsilon
$$

Indeed, from the argument in the proof of [16, Lemma 3.4.10], specifically the computations just prior to the statement of [16, Lemma 3.4.14], we obtain the following estimate. For $K=K(\lambda)$ and $n$ large enough,

$$
E_{0}^{\omega}\left[e^{\lambda H_{n}} \mathbb{1}_{\left\{H_{n}<K n\right\}} \mathbb{1}_{\left\{N^{n}<n^{\delta / 2}\right\}}\right] \geq \frac{1}{2} E_{0}^{\omega}\left[e^{\lambda H_{n}}\right] .
$$

Since

it follows that

$$
1 \leq \frac{E_{0}^{\omega}\left[e^{\lambda H_{n}}\right]}{E_{0}^{\omega}\left[e^{\lambda H_{n}} \mathbb{1}_{\left\{H_{n}<K n\right\}} \mathbb{1}_{\left\{N^{n}<n^{\delta / 2}\right\}}\right]} \leq 2,
$$

$$
\lim _{n \rightarrow \infty} \frac{1}{n}\left(\log E_{0}^{\omega}\left[e^{\lambda H_{n}}\right]-g^{\delta, n}(\omega)\right)=0,
$$

which implies (3.14). Furthermore, since $\lim _{n \rightarrow \infty} \frac{1}{n} \log E_{0}^{\omega}\left[e^{\lambda H_{n}}\right]=\mathcal{J}^{*}(\lambda)$, (3.14) also implies that for $n$ large enough the third term in (3.13) is zero. We conclude that for $n$ large enough,

$$
\mu\left(\omega:\left|\frac{1}{n} \log E_{0}^{\omega}\left[e^{\lambda H_{n}}\right]-\mathcal{J}^{*}(\lambda)\right|>\varepsilon\right) \leq \mu\left(\omega: \frac{1}{n}\left|g^{\delta, n}(\omega)-E_{0}^{\omega}\left[g^{\delta, n}\right]\right|>\frac{1}{3} \varepsilon\right) .
$$

Picking $u=\frac{1}{3} \varepsilon$ in (3.12), we obtain (1.32).

\subsection{Proof of key lemma: concentration on intervals}

\subsubsection{Proof of Lemma 3.1: concentration on half lines}

We first prove (3.1) for the special intervals $(u, 1]$ and $[u, 1]$ with $u \in(0,1)$. Since $\left|Z_{n}\right| \leq n$, this amounts to showing that

$$
\mu\left(\omega:\left|\frac{1}{n} \log P_{0}^{\omega}\left(\frac{Z_{n}}{n} \geq u\right)+\mathcal{I}(u)\right|>\varepsilon\right) \leq C e^{-c n^{1-\delta}},
$$

and

$$
\mu\left(\omega:\left|\frac{1}{n} \log P_{0}^{\omega}\left(\frac{Z_{n}}{n}>u\right)+\mathcal{I}(u)\right|>\varepsilon\right) \leq C e^{-c n^{1-\delta}} .
$$

The proof of (3.20) is analogous to the proof of(3.19). Furthermore, by a reflection argument, we can deduce (3.2) for the intervals $[-1,-u),[-1,-u]$ from (3.19) and (3.20). Indeed, let $\widetilde{\omega}=(\widetilde{\omega}(x))_{x \in \mathbb{Z}}=(1-\omega(-x))_{x \in \mathbb{Z}}$. For $u>0$,

$$
P_{0}^{\omega}\left(\frac{Z_{n}}{n} \leq-u\right)=P_{0}^{\tilde{\omega}}\left(\frac{Z_{n}}{n} \geq u\right) .
$$

Denoting by $\mathcal{I}^{\omega}$ the quenched rate function on $\omega$, we get $\mathcal{I}^{\widetilde{\omega}}(u)=\mathcal{I}^{\omega}(-u)$. Therefore

$$
\frac{1}{n} \log P_{0}^{\omega}\left(\frac{Z_{n}}{n} \leq-u\right)+\mathcal{I}^{\omega}(-u)=\frac{1}{n} \log P_{0}^{\widetilde{\omega}}\left(\frac{Z_{n}}{n} \geq u\right)+\mathcal{I}^{\widetilde{\omega}}(u)
$$

and

$$
\begin{aligned}
& \mu\left(\omega:\left|\frac{1}{n} \log P_{0}^{\omega}\left(\frac{Z_{n}}{n} \leq-u\right)+\mathcal{I}^{\omega}(-u)\right|>\varepsilon\right) \\
& =\mu\left(\omega:\left|\frac{1}{n} \log P_{0}^{\widetilde{\omega}}\left(\frac{Z_{n}}{n} \geq u\right)+\mathcal{I}^{\widetilde{\omega}}(u)\right|>\varepsilon\right) \\
& =\tilde{\mu}\left(\omega:\left|\frac{1}{n} \log P_{0}^{\omega}\left(\frac{Z_{n}}{n} \geq u\right)+\mathcal{I}^{\omega}(u)\right|>\varepsilon\right),
\end{aligned}
$$


RWCRE: ergodic limits and concentration inequalities

where $\tilde{\mu}[\omega \in A]=\mu[\widetilde{\omega} \in A]$ satisfies the conditions of Lemma 3.1. From (3.19) for $\mu$, we obtain (3.19) for $\tilde{\mu}$, which is equivalent to the proof of (3.2) for intervals $[-1,-u$ ). The proof of (3.2) for intervals $[-1,-u]$ is analogous.

To prove (3.20), we derive upper and lower bounds for $\frac{1}{n} \log P_{0}^{\omega}\left(\frac{Z_{n}}{n} \geq u\right)+\mathcal{I}(u)$.

Upper bound. To bound the probabilities on the displacements we can use the hitting times. For $u>0$,

$$
P_{0}^{\omega}\left(\frac{Z_{n}}{n} \geq u\right) \leq P_{0}^{\omega}\left(Z_{n} \geq\lfloor u n\rfloor\right) \leq P_{0}^{\omega}\left(H_{\lfloor u n\rfloor} \leq n\right) .
$$

By the Markov inequality, for $\theta<0$,

$$
P_{0}^{\omega}\left(H_{\lfloor u n\rfloor} \leq n\right) \leq e^{-\theta n} E_{0}^{\omega}\left[e^{\theta H_{\lfloor u n\rfloor}}\right]
$$

and so

$$
\begin{aligned}
\frac{1}{n} \log P_{0}\left(\frac{Z_{n}}{n} \geq u\right) & \leq-\theta+\frac{1}{n} \log E_{0}^{\omega}\left[e^{\theta H_{\lfloor u n\rfloor}}\right] \\
& =-\theta+\frac{1}{n} \log E_{0}^{\omega}\left[e^{\theta H_{\lfloor u n\rfloor}}\right]+u \mathcal{J}^{*}(\theta)-u \mathcal{J}^{*}(\theta) \\
& =-u\left(\theta \frac{1}{u}+O(\omega, u n, \theta)-\mathcal{J}^{*}(\theta)\right)
\end{aligned}
$$

where

$$
O(\omega, \text { un, } \theta):=\mathcal{J}^{*}(\theta)-\frac{1}{u n} \log E_{0}^{\omega}\left[e^{\theta H_{\lfloor u n\rfloor}}\right] .
$$

Taking $\theta<0$ such that $\theta \frac{1}{u}-\mathcal{J}^{*}(\theta)=\mathcal{J}\left(\frac{1}{u}\right)$ (see Fig. 6) and using that $u \mathcal{J}\left(\frac{1}{u}\right)=\mathcal{I}(u)$, we get

$$
\frac{1}{n} \log P_{0}^{\omega}\left(\frac{Z_{n}}{n} \geq u\right)+\mathcal{I}(u) \leq-u O(\omega, u n, \theta) .
$$

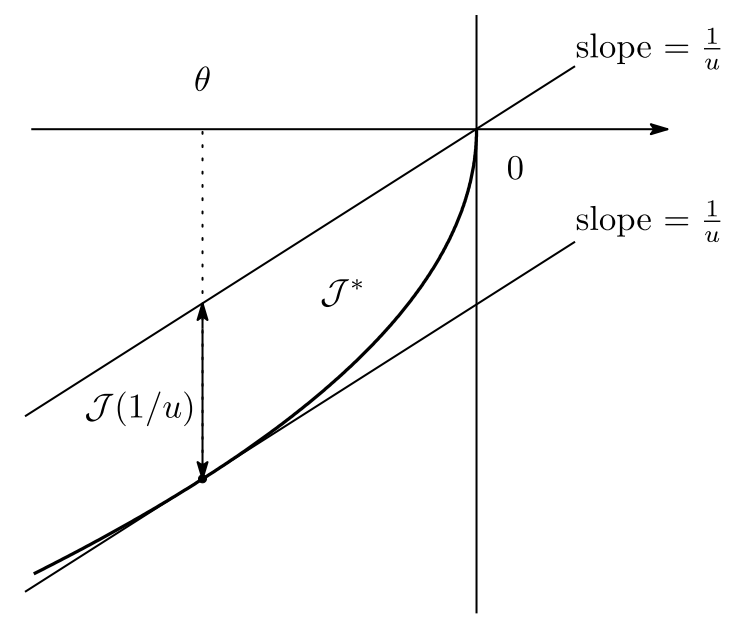

Figure 6: The function $x \mapsto \frac{1}{u} x-\mathcal{J}^{*}(x)$ attains its maximum at $\theta$.

Therefore, using (1.32), we arrive at

$$
\mu\left(\omega: \frac{1}{n} \log P_{0}^{\omega}\left(\frac{Z_{n}}{n} \geq u\right)+\mathcal{I}(u)>\varepsilon\right) \leq \mu(\omega:|O(\omega, u n, \theta)|>\varepsilon)<C e^{-c n^{1-\delta}}
$$


RWCRE: ergodic limits and concentration inequalities

Lower bound. The lower bound for $P_{0}^{\omega}\left(\frac{Z_{n}}{n} \geq u\right)$ is more subtle. Note that, since the steps of the random walk are either +1 or -1 , for $d>0$, we have

$$
n<H_{x}<n+d n \quad \Longrightarrow \quad Z_{n}>x-d n \text {. }
$$

Therefore,

$$
P_{0}^{\omega}\left(\frac{Z_{n}}{n} \geq u\right) \geq P_{0}^{\omega}\left(Z_{n} \geq\lceil u n\rceil\right) \geq P_{0}^{\omega}\left(n \leq H_{\lceil u n\rceil+\lfloor d n\rfloor} \leq n+\lfloor d n\rfloor\right) .
$$

Now, let $m=\lceil u n\rceil+\lfloor d n\rfloor$. Note that if $n \leq H_{m} \leq n+\lfloor d n\rfloor$, then

$$
\frac{1}{u+d+r_{n}} \leq \frac{H_{m}}{m} \leq \frac{1+d+\tilde{r}_{n}}{u+d+r_{n}}
$$

with $r_{n}, \tilde{r}_{n} \rightarrow 0$ as $n \rightarrow 0$. Let $\tilde{d}$ and $\tilde{u}$ be such that

$$
\frac{1}{u+d}<\frac{1}{\tilde{u}}-\tilde{d}<\frac{1}{\tilde{u}}+\tilde{d}<\frac{1+d}{u+d}
$$

Letting $B_{\tilde{d}}\left(\frac{1}{\tilde{u}}\right)$ denote the ball with center $\frac{1}{\tilde{u}}$ and radius $\tilde{d}$, we have, for $n$ large enough,

$$
\frac{1}{n} \log P_{0}^{\omega}\left(\frac{Z_{n}}{n} \geq u\right) \geq \frac{1}{n} \log P_{0}^{\omega}\left(\frac{H_{m}}{m} \in B_{\tilde{d}}\left(\frac{1}{\tilde{u}}\right)\right) .
$$

If $d \rightarrow 0$, then $|\tilde{u}-u| \rightarrow 0$ and $\tilde{d} \rightarrow 0$. Note that $E_{0}^{\omega}\left[e^{\zeta H_{m}}\right]<\infty$ for $\zeta<0$. We define the $\zeta$-tilted probability measure

$$
\frac{\mathrm{d} P_{0}^{\omega, \zeta, m}}{\mathrm{~d} P_{0}^{\omega, m}}(y)=\frac{e^{m \zeta y}}{E_{0}^{\omega}\left[e^{\zeta H_{m}}\right]}, \quad P_{0}^{\omega, m}(\cdot)=P_{0}^{\omega}\left(\frac{H_{m}}{m} \in \cdot\right) .
$$

Recalling that $E_{0}^{\omega}\left[e^{\zeta H_{m}}\right]=\int e^{m \zeta y} \mathrm{~d} P_{0}^{\omega, m}(y)$, we compute

$$
\begin{aligned}
\frac{1}{n} \log P_{0}^{\omega}\left(\frac{H_{m}}{m} \in B_{\tilde{d}}\left(\frac{1}{\tilde{u}}\right)\right) & =\frac{1}{n} \log \int_{B_{\tilde{d}}\left(\frac{1}{\tilde{u}}\right)} \mathrm{d} P_{0}^{\omega, m}(y) \\
& =\frac{1}{n} \log \int_{B_{\tilde{d}\left(\frac{1}{\tilde{u}}\right)}} \frac{E_{0}^{\omega}\left[e^{\zeta H_{m}}\right]}{e^{m \zeta y}} \mathrm{~d} P_{0}^{\omega, \zeta, m}(y) .
\end{aligned}
$$

Now, since $\zeta<0$,

$$
y \in B_{\tilde{d}}\left(\frac{1}{\tilde{u}}\right) \Longrightarrow e^{-m \zeta y} \geq e^{-m \zeta\left(\frac{1}{\tilde{u}}-\tilde{d}\right)} .
$$

Inserting this into (3.35) and replacing $\frac{1}{n} \log E_{0}^{\omega}\left[e^{\zeta H_{m}}\right]$ by $-\frac{m}{n}\left[O(\omega, m, \zeta)-\mathcal{J}^{*}(\zeta)\right]$, yields:

$$
\begin{aligned}
& \frac{1}{n} \log P_{0}^{\omega}\left(\frac{H_{m}}{m} \in B_{\tilde{d}}\left(\frac{1}{\tilde{u}}\right)\right) \\
& \geq \frac{1}{n} \log E_{0}^{\omega}\left[e^{\zeta H_{m}}\right]-\frac{m}{n} \zeta\left(\frac{1}{\tilde{u}}-\tilde{d}\right)+\frac{1}{n} \log P_{0}^{\omega, \zeta, m}\left(B_{\tilde{d}}\left(\frac{1}{\tilde{u}}\right)\right) \\
& =-\hat{u}_{n}\left[\left(\zeta \frac{1}{\hat{u}_{n}}-\mathcal{J}^{*}(\zeta)\right)+O(\omega, m, \zeta)\right]-\hat{d} \zeta+\frac{1}{n} \log P_{0}^{\omega, \zeta, m}\left(B_{\tilde{d}}\left(\frac{1}{\tilde{u}}\right)\right),
\end{aligned}
$$

where $\hat{u}_{n}=\frac{m}{n}$ and $\hat{d}$ is defined by the relation $\frac{m}{n}\left(\frac{1}{\tilde{u}}-\tilde{d}\right)=1+\hat{d}$. Note that

$$
\hat{u}_{n} \rightarrow u, \hat{d} \rightarrow 0 \quad \text { as } \quad d \rightarrow 0, n \rightarrow \infty
$$


RWCRE: ergodic limits and concentration inequalities

Since $\zeta \frac{1}{\hat{u}_{n}}-\mathcal{J}^{*}(\zeta) \leq \mathcal{J}\left(\frac{1}{\hat{u}_{n}}\right)$ and (1.13), combining (3.37) with (3.33) we obtain

$$
\begin{aligned}
\frac{1}{n} \log P_{0}^{\omega}\left(\frac{Z_{n}}{n} \geq u\right)+\mathcal{I}(u) \geq & \mathcal{I}(u)-\mathcal{I}\left(\hat{u}_{n}\right)-\hat{u}_{n} O(\omega, m, \zeta)-\hat{d} \zeta \\
+ & \frac{1}{n} \log P_{0}^{\omega, \zeta, m}\left(B_{\tilde{d}}\left(\frac{1}{\tilde{u}}\right)\right) .
\end{aligned}
$$

Therefore, taking $d$ small enough and $n$ large enough so that

$$
\left|\mathcal{I}(u)-\mathcal{I}\left(\hat{u}_{n}\right)\right|+|\hat{d} \zeta|<\frac{1}{2} \varepsilon,
$$

we get

$$
\begin{aligned}
& \mu\left(\omega: \frac{1}{n} \log P_{0}^{\omega}\left(\frac{Z_{n}}{n} \geq u\right)+\mathcal{I}(u)<-\varepsilon\right) \\
& \leq \mu\left(\omega:-\hat{u}_{n} O(\omega, m, \zeta)+\frac{1}{n} \log P_{0}^{\omega, \zeta, m}\left(B_{\tilde{d}}\left(\frac{1}{\tilde{u}}\right)\right)<-\frac{1}{2} \varepsilon\right) \\
& \leq \mu\left(\omega:-\hat{u}_{n} O(\omega, m, \zeta)<-\frac{1}{4} \varepsilon\right)+\mu\left(\omega: \frac{1}{n} \log P_{0}^{\omega, \zeta, m}\left(B_{\tilde{d}}\left(\frac{1}{\tilde{u}}\right)\right)<-\frac{1}{4} \varepsilon\right) .
\end{aligned}
$$

From (1.32) and the fact that $0<\hat{u}_{n} \leq 1$, it follows that

$$
\mu\left(\omega: \hat{u}_{n}|O(\omega, m, \zeta)|>\frac{1}{4} \varepsilon\right) \leq C e^{-c n^{1-\delta}} .
$$

It therefore remains to prove that

$$
\mu\left(\omega: \frac{1}{n} \log P_{0}^{\omega, \zeta, m}\left(B_{\tilde{d}}\left(\frac{1}{\tilde{u}}\right)\right)<-\frac{1}{4} \varepsilon\right) \leq C e^{-c n^{1-\delta}} .
$$

Let $E_{0}^{\omega, \zeta, m}[f(Y)]$ be expectation of $f$ with respect to $P_{0}^{\omega, \zeta, m}(d Y)$ and $E_{0}^{\omega, m}[f(Y)]$ be expectation of $f$ with respect to $P_{0}^{\omega, m}(d Y)$. Then

$$
E_{0}^{\omega, \zeta, m}\left[e^{m \theta Y}\right]=\frac{E_{0}^{\omega, m}\left[e^{m(\theta+\zeta) Y}\right]}{E_{0}^{\omega, m}\left[e^{m \zeta Y}\right]}=\frac{E_{0}^{\omega}\left[e^{(\theta+\zeta) H_{m}}\right]}{E_{0}^{\omega}\left[e^{\zeta H_{m}}\right]} .
$$

We have

$$
P_{0}^{\omega, \zeta, m}\left(B_{\tilde{d}}\left(\frac{1}{\tilde{u}}\right)^{c}\right)=\underbrace{P_{0}^{\omega, \zeta, m}\left(Y>\frac{1}{\tilde{u}}+\tilde{d}\right)}_{I}+\underbrace{P_{0}^{\omega, \zeta, m}\left(Y<\frac{1}{\tilde{u}}-\tilde{d}\right)}_{I I} .
$$

Since $\mathcal{J}$ is strictly convex in $\frac{1}{\tilde{u}} \in\left(1, \frac{1}{v_{\mu}}\right]$, we can pick $\zeta=\mathcal{J}^{\prime}\left(\frac{1}{\tilde{u}}\right)<0$ to be an exposing plane, i.e., $\zeta$ is such that, for any $y \neq \frac{1}{\tilde{u}}$,

$$
\mathcal{J}(y)-\mathcal{J}\left(\frac{1}{\tilde{u}}\right)>\left(y-\frac{1}{\tilde{u}}\right) \zeta .
$$

We note here that the strict convexity of $\mathcal{J}^{*}$ on $(-\infty, 0]$ implies the strict convexity of $\mathcal{J}$ on $\left(1, \frac{1}{v_{\mu}}\right]$ (recall the relation between $\mathcal{J}$ and $\mathcal{J}^{*}$ in (1.10)). Since $\frac{1}{\tilde{u}}+\tilde{d}>\frac{1}{\tilde{u}}>\frac{1}{\tilde{u}}-\tilde{d}$, we can pick $\theta>0$ and $\sigma<0$ such that (see Fig. 7)

$$
\begin{aligned}
& (\theta+\zeta)\left(\frac{1}{\tilde{u}}+\tilde{d}\right)-\mathcal{J}\left(\frac{1}{\tilde{u}}+\tilde{d}\right)=\mathcal{J}^{*}(\theta+\zeta), \\
& (\sigma+\zeta)\left(\frac{1}{\tilde{u}}-\tilde{d}\right)-\mathcal{J}\left(\frac{1}{\tilde{u}}-\tilde{c}\right)=\mathcal{J}^{*}(\sigma+\zeta)
\end{aligned}
$$


RWCRE: ergodic limits and concentration inequalities
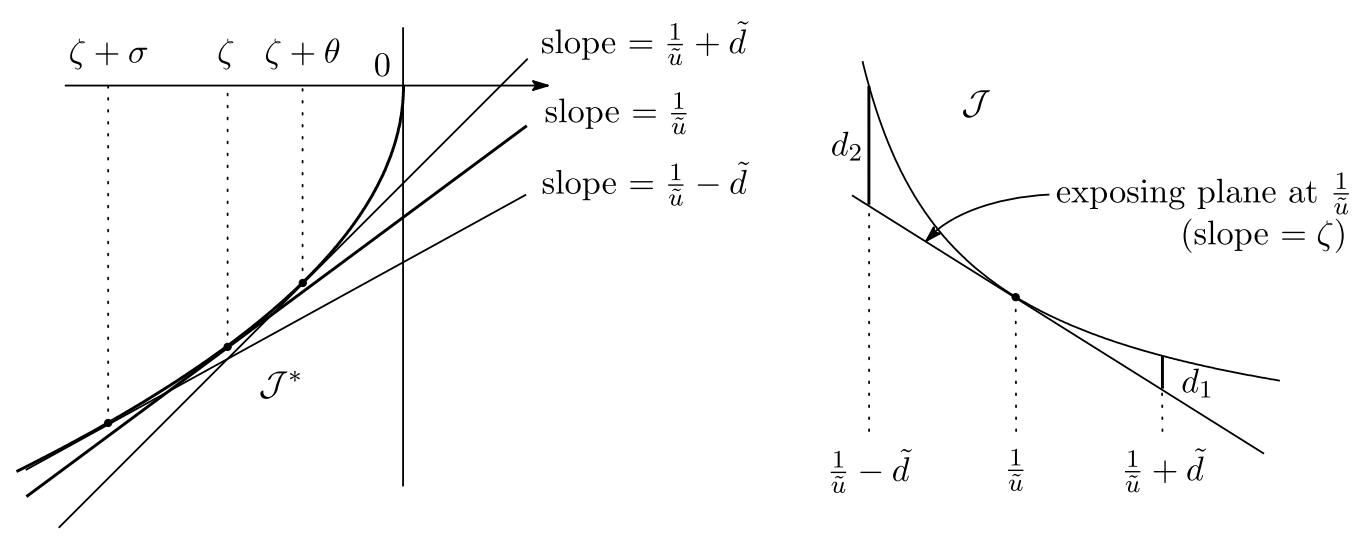

Figure 7: Left: The graph shows the implicit relation between $\mathcal{J}$ and $\mathcal{J}^{*}$ : for $x<v_{\mu}^{-1}$, $\mathcal{J}(x)=x y-\mathcal{J}^{*}(y)$ with $x=\left(\mathcal{J}^{*}\right)^{\prime}(y)$. The equations in (3.47) follow from this relation. Right: The exposing plane condition shows that $d_{1}$ in (3.49) and $d_{2}$ in (3.52) are strictly positive.

Bound for $I$. For $\theta>0$,

$$
\begin{aligned}
& P_{0}^{\omega, \zeta, m}\left(Y>\frac{1}{\tilde{u}}+\tilde{d}\right) \leq e^{-m \theta\left(\frac{1}{\tilde{u}}+\tilde{d}\right)} E_{0}^{\omega, \zeta, m}\left[e^{\theta Y}\right] \\
& =\exp \left\{m\left[-\theta\left(\frac{1}{\tilde{u}}+\tilde{d}\right)+\frac{1}{m}\left(\log E_{0}^{\omega}\left[e^{(\theta+\zeta) H_{m}}\right]-\log E_{0}^{\omega}\left[e^{\zeta H_{m}}\right]\right)\right]\right\} \\
& \leq \exp \left\{m\left[-\theta\left(\frac{1}{\tilde{u}}+\tilde{d}\right)+\mathcal{J}^{*}(\theta+\zeta)-\mathcal{J}^{*}(\zeta)-O(\omega, m, \theta+\zeta)+O(\omega, m, \zeta)\right]\right\} .
\end{aligned}
$$

By (3.46) with $y=\frac{1}{\tilde{u}}+\tilde{d}$, we find that

$$
\begin{aligned}
& -\theta\left(\frac{1}{\tilde{u}}+\tilde{d}\right)+\mathcal{J}^{*}(\theta+\zeta)-\mathcal{J}^{*}(\zeta) \\
& =-(\theta+\zeta)\left(\frac{1}{\tilde{u}}+\tilde{d}\right)+\mathcal{J}^{*}(\theta+\zeta)-\mathcal{J}^{*}(\zeta)+\zeta\left(\frac{1}{\tilde{u}}+\tilde{d}\right) \\
& =-\mathcal{J}\left(\frac{1}{\tilde{u}}+\tilde{d}\right)+\zeta\left(\frac{1}{\tilde{u}}+\tilde{d}\right)-\mathcal{J}^{*}(\zeta) \\
& \leq-\mathcal{J}\left(\frac{1}{\tilde{u}}+\tilde{d}\right)+\zeta\left(\frac{1}{\tilde{u}}+\tilde{d}\right)-\zeta \frac{1}{\tilde{u}}+\mathcal{J}\left(\frac{1}{\tilde{u}}\right)=-d_{1}<0
\end{aligned}
$$

(see Fig. 7). On the set $A_{I}=\left\{\omega:|O(\omega, m, \theta+\zeta)-O(\omega, m, \zeta)|<\frac{1}{2}\left|d_{1}\right|\right\}$, we have

$$
P_{0}^{\omega, \zeta, m}\left(Y>\frac{1}{\tilde{u}}+\tilde{d}\right)<e^{-m \frac{\left|d_{1}\right|}{2}} \rightarrow 0 .
$$

Bound for $I I$. Again, for $\sigma<0$,

$$
\begin{aligned}
& P_{0}^{\omega, \zeta, m}\left(Y<\frac{1}{\tilde{u}}-\tilde{d}\right) \leq e^{-m \sigma\left(\frac{1}{\tilde{u}}-\tilde{d}\right)} E_{0}^{\omega, \zeta, m}\left[e^{\sigma Y}\right] \\
& =\exp \left\{m\left[-\sigma\left(\frac{1}{\tilde{u}}-\tilde{d}\right)+\frac{1}{m}\left(\log E_{0}^{\omega}\left[e^{(\sigma+\zeta) H_{m}}\right]-\log E_{0}^{\omega}\left[e^{\zeta H_{m}}\right]\right)\right]\right\} \\
& \leq \exp \left\{m\left[-\sigma\left(\frac{1}{\tilde{u}}-\tilde{d}\right)+\mathcal{J}^{*}(\sigma+\zeta)-\mathcal{J}^{*}(\zeta)-O(\omega, m, \sigma+\zeta)+O(\omega, m, \zeta)\right]\right\} .
\end{aligned}
$$


RWCRE: ergodic limits and concentration inequalities

Similarly to (3.49), using (3.46) with $y=\frac{1}{\tilde{u}}-\tilde{d}$, we obtain

$$
-\sigma\left(\frac{1}{\tilde{u}}-\tilde{d}\right)+\mathcal{J}^{*}(\sigma+\zeta)-\mathcal{J}^{*}(\zeta)=-d_{2}<0
$$

(see Fig 7). For $\omega \in A_{I I}=\left\{\omega:|O(\omega, m, \sigma+\zeta)-O(\omega, m, \zeta)|<\frac{1}{2}\left|d_{2}\right|\right\}$,

$$
P_{0}^{\omega, \zeta, m}\left(Y<\frac{1}{\tilde{u}}-\tilde{d}\right)<e^{-m \frac{1}{2}\left|d_{2}\right|} \rightarrow 0 .
$$

Conclusion. For $n$ large enough, using (3.50) and (3.53) we see that

$$
\omega \in A_{I} \cap A_{I I} \quad \Longrightarrow \quad P_{0}^{\omega, \zeta, m}\left(B_{\tilde{d}}\left(\frac{1}{\tilde{u}}\right)^{c}\right)<\frac{1}{2} \quad \Longrightarrow \quad P_{0}^{\omega, \zeta, m}\left(B_{\tilde{d}}\left(\frac{1}{\tilde{u}}\right)\right) \geq \frac{1}{2}
$$

and therefore, for large $n$, we conclude that

$$
\left|\frac{1}{n} \log P_{0}^{\omega, \zeta, m}\left(B_{\tilde{d}}\left(\frac{1}{\tilde{u}}\right)\right)\right|<\frac{1}{n} \log 2<\frac{1}{4} \varepsilon .
$$

Hence

$$
\mu\left(\omega: \frac{1}{n} \log P_{0}^{\omega, \zeta, m}\left(B_{\tilde{d}}\left(\frac{1}{\tilde{u}}\right)\right)<-\frac{1}{4} \varepsilon\right) \leq \mu\left(A_{I}^{c}\right)+\mu\left(A_{I I}^{c}\right) .
$$

To complete the proof, note that (1.32) implies

$$
\mu\left(A_{I}^{c}\right)+\mu\left(A_{I I}^{c}\right) \leq C e^{-c n^{1-\delta}} .
$$

It is worth mentioning that the constant in (3.57) does not depends on $n$. For fixed $u$ we choose $\varepsilon>0$, and (3.40) together with (3.38) gives us $d, \tilde{d}$ and $\tilde{u}$. After that, $d_{1}, d_{2}$ are given by the exposing plane conditions at the boundary of the ball of radius $\tilde{d}$ centered at $\frac{1}{\tilde{u}}$ (see (3.49) and (3.52)). Thus, even though the constant in (3.57) depends on $d_{1}, d_{2}$, the latter are functions of $u$ and $\varepsilon$ only, and not of $n$. The latter estimate, together with the bounds in (3.41) and (3.42), yield

$$
\mu\left(\omega: \frac{1}{n} \log P_{0}^{\omega}\left(\frac{Z_{n}}{n} \geq u\right)+\mathcal{I}(u)<-\varepsilon\right)<C e^{-c n^{1-\delta}} .
$$

Therefore (3.20) in Lemma 3.1 follows from (3.28) and (3.58).

\subsubsection{Proof of Lemma 3.1: general intervals}

We will split the proof for general $\Delta=(a, b]$ in two cases. The proof for $\Delta=[a, b]$ is similar.

Case $0 \leq a$. We start from the equation

$$
P_{0}^{\omega}\left(\frac{Z_{n}}{n} \in \Delta\right)=P_{0}^{\omega}\left(\frac{Z_{n}}{n}>a\right)-P_{0}^{\omega}\left(\frac{Z_{n}}{n}>b\right) .
$$

Define $e(\omega, u, n):=\frac{1}{n} \log P_{0}^{\omega}\left(\frac{Z_{n}}{n}>u\right)+\mathcal{I}(u)$. Since $\mathcal{I}(b)-\mathcal{I}(a)=\eta>0$, we obtain

$$
\frac{P_{0}^{\omega}\left(\frac{Z_{n}}{n}>a\right)}{P_{0}^{\omega}\left(\frac{Z_{n}}{n}>b\right)}=e^{n(\mathcal{I}(b)-\mathcal{I}(a)+e(\omega, a, n)-e(\omega, b, n))}=e^{n(\eta+e(\omega, a, n)-e(\omega, b, n))} .
$$

When $|e(\omega, a, n)-e(\omega, b, n)|<\frac{1}{2} \eta$, we have

$$
P_{0}^{\omega}\left(\frac{Z_{n}}{n}>b\right) \leq e^{-n \frac{\eta}{2}} P_{0}^{\omega}\left(\frac{Z_{n}}{n}>a\right) .
$$


For large enough $n$, as soon as $e^{-n \frac{\eta}{2}}<\frac{1}{2}$ we get

$$
\frac{1}{2} P_{0}^{\omega}\left(\frac{Z_{n}}{n}>a\right) \leq P_{0}^{\omega}\left(\frac{Z_{n}}{n} \in \Delta\right) \leq P_{0}^{\omega}\left(\frac{Z_{n}}{n}>a\right)
$$

which implies that

$$
\left|\frac{1}{n} \log P_{0}^{\omega}\left(\frac{Z_{n}}{n} \in \Delta\right)-\frac{1}{n} \log P_{0}^{\omega}\left(\frac{Z_{n}}{n}>a\right)\right|<\frac{1}{n} \log 2 .
$$

Therefore

$$
\begin{aligned}
& \mu\left(\omega:\left|\frac{1}{n} \log P_{0}^{\omega}\left(\frac{Z_{n}}{n} \in \Delta\right)-\frac{1}{n} \log P_{0}^{\omega}\left(\frac{Z_{n}}{n}>a\right)\right|>\frac{1}{n} \log 2\right) \\
& \leq \mu\left(\omega:|e(\omega, a, n)-e(\omega, b, n)|>\frac{1}{2} \eta\right) \\
& \leq \mu\left(\omega:|e(\omega, a, n)|>\frac{1}{4} \eta\right)+\mu\left(\omega:|e(\omega, b, n)|>\frac{1}{4} \eta\right) .
\end{aligned}
$$

Since the concentration (3.20) in Lemma 3.1 bounds both terms in (3.64), after we pick $n$ large enough so that $\frac{\log 2}{n}<\varepsilon$, we obtain (3.1).

Case $b \leq 0$. In this case we have the following equation:

$$
P_{0}^{\omega}\left(\frac{Z_{n}}{n} \in \Delta\right)=P_{0}^{\omega}\left(\frac{Z_{n}}{n} \leq b\right)-P_{0}^{\omega}\left(\frac{Z_{n}}{n} \leq a\right) .
$$

Similarly, we define $\tilde{e}(\omega, u, n):=\frac{1}{n} \log P_{0}^{\omega}\left(\frac{Z_{n}}{n} \leq u\right)+\mathcal{I}(u)$. Since $\mathcal{I}(a)-\mathcal{I}(b)=\eta>0$, we obtain

$$
\frac{P_{0}^{\omega}\left(\frac{Z_{n}}{n} \leq b\right)}{P_{0}^{\omega}\left(\frac{Z_{n}}{n} \leq a\right)}=e^{n(\mathcal{I}(a)-\mathcal{I}(b)-\tilde{e}(\omega, a, n)+\tilde{e}(\omega, b, n))}=e^{n(\eta+\tilde{e}(\omega, a, n)-\tilde{e}(\omega, b, n))} .
$$

When $|\tilde{e}(\omega, a, n)-\tilde{e}(\omega, b, n)|<\frac{1}{2} \eta$,

$$
P_{0}^{\omega}\left(\frac{Z_{n}}{n} \leq a\right) \leq e^{-n \frac{\eta}{2}} P_{0}^{\omega}\left(\frac{Z_{n}}{n} \leq b\right)
$$

As we did in (3.61)-(3.63), for large $n$ as soon as $e^{-n \frac{\eta}{2}}<\frac{1}{2}$ we conclude that

$$
\begin{aligned}
& \mu\left(\omega:\left|\frac{1}{n} \log P_{0}^{\omega}\left(\frac{Z_{n}}{n} \in \Delta\right)-\frac{1}{n} \log P_{0}^{\omega}\left(\frac{Z_{n}}{n} \leq b\right)\right|>\frac{1}{n} \log 2\right) \\
& \leq \mu\left(\omega:|\tilde{e}(\omega, a, n)-\tilde{e}(\omega, b, n)|>\frac{1}{2} \eta\right) \\
& \leq \mu\left(\omega:|\tilde{e}(\omega, a, n)|>\frac{1}{4} \eta\right)+\mu\left(\omega:|\tilde{e}(\omega, b, n)|>\frac{1}{4} \eta\right) .
\end{aligned}
$$

Since the concentration (3.20) in Lemma 3.1 bounds both terms in (3.68), after we pick $n$ large enough so that $\frac{\log 2}{n}<\varepsilon$, we obtain (3.2) and Lemma 3.1 follows.

\subsection{Concentration of cumulants}

In this section we prove (1.33)

Note that $\frac{Z_{n}}{n} \in[-1,1]$. Consider the following block decomposition (see Fig. 8):

$$
\Delta_{i}^{N}= \begin{cases}{\left[-1,-1+\frac{1}{N}\right],} & \text { if } i=-N \\ \left(\frac{i}{N}, \frac{i+1}{N}\right], & \text { if } i \in\{-N+1, \ldots, N-1\}\end{cases}
$$


RWCRE: ergodic limits and concentration inequalities

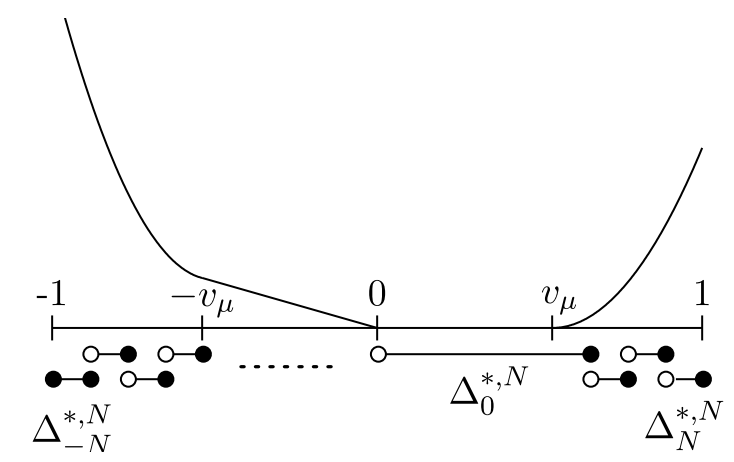

Figure 8: Block decomposition of $[-1,1]$. Black and white circles indicate closed and open boundaries of the intervals, respectively. All the intervals are of length $\frac{1}{N}$, except possibly $\Delta_{0}^{*, N}$, which contains the flat piece $\left(0, v_{\mu}\right]$.

To deal with the flat piece of the rate function $\mathcal{I}$ in the positive-speed case, we define the following interval $\Delta_{0}^{*, N}$ containing $\left(0, v_{\mu}\right]$ (see Fig. 8):

$$
\Delta_{0}^{*, N}=\left(0, \frac{\left\lfloor v_{\mu} N+1\right\rfloor}{N}\right]=\bigcup_{i=-N}^{N-1}\left\{\Delta_{i}^{N}: \mathcal{I}\left(\frac{i-1}{N}\right)=0\right\}, \quad \Delta_{i}^{*, N}=\Delta_{i}^{N} \backslash \Delta_{0}^{*, N} .
$$

By the intermediate value theorem, we have

$$
E_{0}^{\omega}\left[e^{n \lambda \frac{Z_{n}}{n}} \mathbb{1}_{\left\{\frac{Z_{n}}{n} \in \Delta_{i}^{*, N}\right\}}\right]=e^{n \lambda u_{i}^{*}} P_{0}^{\omega}\left(\frac{Z_{n}}{n} \in \Delta_{i}^{*, N}\right)
$$

for some $u_{i}^{*} \in \Delta_{i}^{*, N}$. Now,

$$
\begin{aligned}
\frac{1}{n} \log E_{0}^{\omega}\left[e^{\lambda Z_{n}}\right]-\mathcal{I}^{*}(\lambda) & =\frac{1}{n} \log e^{-n \mathcal{I}^{*}(\lambda)} \sum_{i=-N}^{N-1} E_{0}^{\omega}\left[e^{n \lambda \frac{Z_{n}}{n}} \mathbb{1}_{\left\{\frac{Z_{n}}{n} \in \Delta_{i}^{*, N}\right\}}\right] \\
& =\frac{1}{n} \log \sum_{i=-N}^{N-1} e^{n \lambda u_{i}^{*}-n \mathcal{I}_{n}^{\omega}\left(\Delta_{i}^{*, N}\right)-n \mathcal{I}^{*}(\lambda)}
\end{aligned}
$$

where $\mathcal{I}_{n}^{\omega}(\Delta):=-\frac{1}{n} \log P_{0}^{\omega}\left(\frac{Z_{n}}{n} \in \Delta\right)$. Since $\mathcal{I}_{n}^{\omega}(\Delta)$ converges to $\mathcal{I}(\Delta)=\inf _{x \in \Delta} \mathcal{I}(x)$ as $n \rightarrow \infty$, we define the block error

$$
o(n, \Delta, \omega):=\mathcal{I}(\Delta)-\mathcal{I}_{n}^{\omega}(\Delta)
$$

and obtain

$$
\frac{1}{n} \log E_{0}^{\omega}\left[e^{\lambda Z_{n}}\right]-\mathcal{I}^{*}(\lambda)=\frac{1}{n} \log \sum_{i=-N}^{N-1} e^{n\left(\lambda u_{i}^{*}-\mathcal{I}\left(\Delta_{i}^{*, N}\right)-\mathcal{I}^{*}(\lambda)+o\left(n, \Delta_{i}^{*, N}, \omega\right)\right)} .
$$

To estimate (3.74), we will need the following lemma.

\section{Lemma 3.2 (Reduction to the worst block).}

Given $\lambda \in \mathbb{R}$ and $\varepsilon>0$, there is an $N_{0}$ such that, for $N>N_{0}$ and $n>n_{0}(N)$,

$$
\left|\frac{1}{n} \log E_{0}^{\omega}\left[e^{\lambda Z_{n}}\right]-\mathcal{I}^{*}(\lambda)\right| \leq \frac{1}{2} \varepsilon+\max _{-N \leq i \leq N-1}\left|o\left(n, \Delta_{i}^{*, N}, \omega\right)\right|
$$

with $o\left(n, \Delta_{i}^{*, N}, \omega\right)$ as in (3.73). 
RWCRE: ergodic limits and concentration inequalities

Proof. If $\lambda=0$, then the left-hand side of (3.75) is equal to zero. Therefore we focus on the case $\lambda \neq 0$. Since $\mathcal{I}$ is uniformly continuous in $[-1,1]$, we have

$$
\delta_{N}=\sup _{|s-t| \leq 1 / N}|\mathcal{I}(s)-\mathcal{I}(t)| \rightarrow 0 \quad \text { as } N \rightarrow \infty .
$$

Let $\delta_{i}=\mathcal{I}\left(u_{i}^{*}\right)-\mathcal{I}\left(\Delta_{i}^{*, N}\right)$, and note that $0 \leq \delta_{i} \leq \delta_{N}$.

Upper bound. Since

$$
\mathcal{I}^{*}(\lambda)=\sup _{u \in \mathbb{R}}[\lambda u-\mathcal{I}(u)] \geq \lambda u_{i}^{*}-\mathcal{I}\left(u_{i}^{*}\right)
$$

we get the bound

$$
\lambda u_{i}^{*}-\mathcal{I}\left(\Delta_{i}^{*, N}\right)-\mathcal{I}^{*}(\lambda) \leq \lambda u_{i}^{*}-\left[\mathcal{I}\left(u_{i}^{*}\right)-\delta_{i}\right]-\left[\lambda u_{i}^{*}-\mathcal{I}\left(u_{i}^{*}\right)\right]=\delta_{i} \leq \delta_{N} .
$$

Let $N_{0}$ be such that $\delta_{N_{0}}<\frac{1}{2} \varepsilon$. For $N>N_{0}$, let $n_{0}(N)$ be such that $\delta_{N_{0}}+\frac{\log 2 N}{n_{0}}<\frac{1}{2} \varepsilon$. For $n>n_{0}(N)$, we have

$$
\begin{aligned}
\frac{1}{n} \log E_{0}^{\omega}\left[e^{\lambda Z_{n}}\right]-\mathcal{I}^{*}(\lambda) & \leq \frac{1}{n} \log \sum_{i=-N}^{N-1} e^{n\left(\delta_{N}+o\left(n, \Delta_{i}^{*, N}, \omega\right)\right)} \\
& \leq \delta_{N}+\frac{\log 2 N}{n}+\max _{-N \leq i \leq N-1} o\left(n, \Delta_{i}^{*, N}, \omega\right) \\
& \leq \frac{1}{2} \varepsilon+\max _{-N \leq i \leq N-1} o\left(n, \Delta_{i}^{*, N}, \omega\right) .
\end{aligned}
$$

Lower bound. Let $\hat{x}$ be such that $\mathcal{I}^{*}(\lambda)=\lambda \hat{x}-\mathcal{I}(\hat{x})$. Then there exists a $\hat{\imath}$ such that $\hat{x} \in \Delta_{\hat{\imath}}^{*, N}$. For large $N$, we see that $\hat{x} \notin \Delta_{0}^{*, N}$. Indeed, if $\lambda<0$, then $\hat{x} \leq 0$ and therefore $\hat{x} \notin \Delta_{0}^{*, N}$. On the other hand, if $\lambda>0$, then $\hat{x}>v_{\mu}$. Pick $N$ large enough so that $v_{\mu}+\frac{1}{N}<\hat{x}$. Since $\Delta_{0}^{*, N} \subset\left(0, v_{\mu}+\frac{1}{N}\right]$, we conclude that $\hat{x} \notin \Delta_{0}^{*, N}$.

Since $\hat{x} \in \Delta_{\hat{\iota}}^{*, N}$, it follows that $\left|\hat{x}-u_{\hat{\iota}}^{*}\right|<\frac{1}{N}$. Choosing $N_{0}$ so that $\frac{|\lambda|}{N_{0}}+\delta_{N_{0}}<\frac{1}{2} \varepsilon$, we obtain

$$
\begin{aligned}
\frac{1}{n} \log E_{0}^{\omega}\left[e^{\lambda Z_{n}}\right]-\mathcal{I}^{*}(\lambda) & \geq \frac{1}{n} \log e^{n\left(\left[\lambda u_{\hat{\iota}}^{*}-\mathcal{I}\left(\Delta_{\hat{\iota}}^{*, N}\right)\right]-[\lambda \hat{x}-\mathcal{I}(\hat{x})]+o\left(n, \Delta_{\hat{\iota}}^{*, N}, \omega\right)\right)} \\
& \geq-\left(\frac{|\lambda|}{N}+\delta_{N}\right)+o\left(n, \Delta_{\hat{\iota}}^{*, N}, \omega\right) \\
& \geq-\frac{1}{2} \varepsilon+o\left(n, \Delta_{\hat{\iota}}^{*, N}, \omega\right)
\end{aligned}
$$

The claim in (3.75) follows from (3.79) and (3.80).

In view of Lemma 3.2, we can bound

$$
\begin{aligned}
\mu\left(\omega:\left|\frac{1}{n} \log E_{0}^{\omega}\left[e^{\lambda Z_{n}}\right]-\mathcal{I}^{*}(\lambda)\right|>\varepsilon\right) & \leq \mu\left(\omega: \max _{-N \leq i \leq N-1}\left|o\left(n, \Delta_{i}^{*, N}, \omega\right)\right|>\frac{1}{2} \varepsilon\right) \\
& \leq \sum_{i=-N}^{N-1} \mu\left(\omega:\left|o\left(n, \Delta_{i}^{*, N}, \omega\right)\right|>\frac{1}{2} \varepsilon\right) .
\end{aligned}
$$

Since there are only finitely many terms in the sum, (1.33) follows from Lemma 3.1. 
RWCRE: ergodic limits and concentration inequalities

\subsection{Concentration of displacements}

The proof of (1.34) follows from Lemma 3.1 and from the uniform continuity of $\mathcal{I}$ as stated in (3.76). Indeed, for $\varepsilon>0$, let $N$ be such that $\delta_{N}<\frac{1}{3} \varepsilon$. For $x \geq 0$ let $\Delta_{i}^{N}$ in (3.69) be such that $x \in \Delta_{i}^{N} \subset[a, b]$ with $b-a=\frac{1}{N}$. Remember the notation $[[n x]]$ given before the statement of Theorem 1.13. For $n$ sufficiently large so that $[n x]>[n a]$ we have the following upper bound

$$
\begin{aligned}
\frac{1}{n} \log P_{0}^{\omega}\left(Z_{n}=[[n x]]\right)+\mathcal{I}(u) & \leq \frac{1}{n} \log P_{0}^{\omega}\left(\frac{Z_{n}}{n} \in[a, b]\right)+\mathcal{I}(a)+|\mathcal{I}(u)-\mathcal{I}(a)| \\
& \leq \frac{1}{n} \log P_{0}^{\omega}\left(\frac{Z_{n}}{n} \in[a, b]\right)+\mathcal{I}(a)+\frac{1}{3} \varepsilon
\end{aligned}
$$

Using (3.1) with $\frac{2}{3} \varepsilon$ we obtain that

$$
\begin{aligned}
& \mu\left(\omega: \frac{1}{n} \log P_{0}^{\omega}\left(Z_{n}=[[n x]]\right)+\mathcal{I}(x)>\varepsilon\right) \\
& \leq \mu\left(\omega:\left|\frac{1}{n} \log P_{0}^{\omega}\left(\frac{Z_{n}}{n}>a\right)+\mathcal{I}(a)\right|>\frac{2}{3} \varepsilon\right) \leq C e^{-c n^{1-\delta}} .
\end{aligned}
$$

The lower bound is more delicate. By the uniform ellipticity of the environment, for $y \in[n a, n b] \cap \mathbb{Z}$ we can estimate

$$
P_{0}^{\omega}\left(Z_{n-n(b-a)}=y\right) \mathfrak{c}^{n(b-a)} \leq P_{0}^{\omega}\left(Z_{n}=[[n x]]\right),
$$

from which:

$$
\begin{aligned}
P_{0}^{\omega}\left(Z_{n-n(b-a)} \in[n a, n b]\right) & =\sum_{y \in[n a, n b] \cap \mathbb{Z}} P_{0}^{\omega}\left(Z_{n-n(b-a)}=y\right) \\
& \leq(n(b-a)+1) P_{0}^{\omega}\left(Z_{n}=[[n x]]\right) \mathfrak{c}^{-n(b-a)} .
\end{aligned}
$$

Set next $n^{\prime}=n(1-(b-a)), \tilde{a}=\frac{a}{1-(b-a)}, \tilde{b}=\frac{b}{1-(b-a)}$, and take $\frac{1}{n} \log$ on both sides of (3.85) to obtain

$$
\begin{aligned}
\frac{1}{n} \log P_{0}^{\omega}\left(Z_{n}=[[n x]]\right) & \geq \frac{1}{n} \log P_{0}^{\omega}\left(\frac{Z_{n^{\prime}}}{n^{\prime}} \in[\tilde{a}, \tilde{b}]\right) \\
& -(b-a)|\log \mathfrak{c}|-\frac{1}{n} \log (n(b-a)+1) .
\end{aligned}
$$

Since $b-a=\frac{1}{N}$, we can take $N, n$ sufficiently large so that

$$
|(b-a)| \log \mathfrak{c}\left|+\frac{1}{n} \log (2 n+1)(b-a)\right|<\frac{1}{3} \varepsilon,
$$

and

$$
|(1-(b-a)) \mathcal{I}(\tilde{a})-\mathcal{I}(x)|<\frac{1}{3} \varepsilon,
$$

which together with (3.86) yields to

$$
\begin{aligned}
& \frac{1}{n} \log P_{0}^{\omega}\left(Z_{n}=[[n x]]\right)+\mathcal{I}(x) \\
& \quad \geq(1-(b-a)) \frac{1}{n^{\prime}} \log P_{0}^{\omega}\left(\frac{Z_{n^{\prime}}}{n^{\prime}} \in[\tilde{a}, \tilde{b}]\right)+\mathcal{I}(x)-\frac{1}{3} \varepsilon .
\end{aligned}
$$

Using (3.1) with $\frac{1}{3} \varepsilon$ we obtain that

$$
\begin{aligned}
& \mu\left(\omega: \frac{1}{n} \log P_{0}^{\omega}\left(Z_{n}=[[n x]]\right)+\mathcal{I}(x)<-\varepsilon\right) \\
& \leq \mu\left(\omega:(1-(b-a))\left|\frac{1}{n^{\prime}} \log P_{0}^{\omega}\left(\frac{Z_{n^{\prime}}}{n^{\prime}} \in[\tilde{a}, \tilde{b}]\right)+\mathcal{I}(\tilde{a})\right|>\frac{1}{3} \varepsilon\right) \leq C e^{-c n^{1-\delta}} .
\end{aligned}
$$


Putting (3.90) and (3.83) together, for large enough $N$ and $0 \leq a \leq x \leq b=a+\frac{1}{N}$ we obtain

$$
\mu\left(\omega: \sup _{x \in[a, b]}\left|\frac{1}{n} \log P_{0}^{\omega}\left(Z_{n}=[[n x]]\right)+\mathcal{I}(x)\right|>\varepsilon\right) \leq C e^{-c n^{1-\delta}} .
$$

Similarly, by (3.2), for large enough $N$ and $a=b-\frac{1}{N} \leq x \leq b \leq 0$ :

$$
\mu\left(\omega: \sup _{x \in[a, b]}\left|\frac{1}{n} \log P_{0}^{\omega}\left(Z_{n}=[[n x]]\right)+\mathcal{I}(x)\right|>\varepsilon\right) \leq C e^{-c n^{1-\delta}} .
$$

To conclude the proof of (1.34), it suffices to note that

$$
\begin{aligned}
& \mu\left(\omega: \sup _{x \in[-1,1]}\left|\frac{1}{n} \log P_{0}^{\omega}\left(Z_{n}=[[n x]]\right)+\mathcal{I}(x)\right|>\varepsilon\right) \\
& \leq \sum_{i=-N}^{N} \mu\left(\omega: \sup _{x \in \Delta_{i}^{N}}\left|\frac{1}{n} \log P_{0}^{\omega}\left(Z_{n}=[n x]\right)+\mathcal{I}(x)\right|>\varepsilon\right) \leq C e^{c n^{1-\delta}} .
\end{aligned}
$$

\section{Proofs of SLLN and LDP}

\subsection{Proof of SLLN}

In this section we prove Theorem 1.10. We will prove the SLLN under the annealed law. After that we get Theorem 1.10 by noting that, for any event $A$, if $P_{0}^{\mu, \tau}(A)=1$, then $P_{0}^{\Omega, \tau}(A)=1$ for $\mu^{\mathbb{N}}$-a.e. $\Omega$, and taking $A=\left\{\lim _{n \rightarrow \infty} \frac{X_{n}}{n}=v_{\mu}\right\}$ to get the claim.

To prove the SLLN under the annealed law, we will use Theorem 1.12. To this aim, let $\mathbb{P}$ be the joint law of doubly indexed variables $\psi_{n}^{(k)}$ that are pair-wise independent in $k$ and such that, for each $k, \psi_{n}^{(k)}$ has law $P_{0}^{\mu}\left(Z_{n}=\cdot\right)$. From (1.26) we see that $\psi_{T_{k}}^{(k)}$ is distributed as $Y_{k}$ and $\psi_{\bar{T}^{n}}^{(\ell(n))}$ is distributed as $\bar{Y}^{n}$.

Assumptions (A1) and (A2) are trivially satisfied. It remains to check (1.29) with $L=v_{\mu}$, for which we use the annealed large deviation estimates for RWRE. In fact, from Proposition 1.6 we get

$$
\limsup _{n \rightarrow \infty} \frac{1}{n} \log P_{0}^{\mu}\left(\left|\frac{Z_{n}}{n}-v_{\mu}\right| \geq \varepsilon\right) \leq-\mathcal{I}\left(v_{\mu}+\varepsilon\right) \vee-\mathcal{I}\left(v_{\mu}-\varepsilon\right) .
$$

In the zero-speed case, since $-\mathcal{I}\left(v_{\mu}+\varepsilon\right) \vee-\mathcal{I}\left(v_{\mu}-\varepsilon\right)<0$, the speed of decay is exponential in $n$ and (A3) holds. In the positive-speed case, $\mathcal{I}\left(v_{\mu}-\varepsilon\right)=0$ and the bound in (4.1) is not useful. However, Proposition 1.7 yields the following bound in the flat piece:

$$
\limsup _{n \rightarrow \infty} \frac{1}{\log n} \log P_{0}^{\mu}\left(\left|\frac{Z_{n}}{n}\right|<v_{\mu}-\varepsilon\right)<0 .
$$

Since (1.31) holds and (4.2) implies (1.29) with $\delta>0$. By (1.30), Theorem 1.10 follows.

\subsection{Proof of LDP}

In this section we prove Theorem 1.11. We will first prove existence of the rate function, and afterwards prove a large deviation principle with respect to this rate function. It is worth noticing that if the measure on the environment process is stationary and ergodic with respect to the family of space-time translations, then the rate function can be obtained by using Liggett's subadditive ergodic theorem [12] (see [5] for this approach). In our case, the environment process is not stationary with respect to time translations and therefore we need an ad hoc argument that will combine the specific concentration properties of RWCRE of Theorem 1.13 with the concentration estimates in Theorem 1.12. We start with two lemmas that are instrumental for our argument. 
RWCRE: ergodic limits and concentration inequalities

Lemma 4.1. Let $|z| \leq 1$ and $|x|<1$. Then, for each $n \in \mathbb{N}_{0}$,

$$
-\log P_{0}^{\Omega, \tau}\left(X_{n^{\prime}}=\left[\left[n^{\prime} x\right]\right]\right) \leq-\log P_{0}^{\Omega, \tau}\left(X_{n}=[[n z]]\right)-\left(n^{\prime}-n\right) \log \mathfrak{c}
$$

with $n^{\prime}:=n+2 n \frac{|x-z|}{1-|x|}$.

Proof. Since $\left|n^{\prime} x-n z\right| \leq n^{\prime}-n$, the Chapman-Kolmogorov equation combined with the uniform ellipticity (1.5) yields

$$
P_{0}^{\Omega, \tau}\left(X_{n^{\prime}}=\left[\left[n^{\prime} x\right]\right]\right) \geq P_{0}^{\Omega, \tau}\left(X_{n}=[[n z]]\right) \mathfrak{c}^{n^{\prime}-n} .
$$

Take $-\frac{1}{n} \log$ on both sides.

The next lemma, a convergence result for the cumulants of the displacements of RWCRE, will be used in the lower bound that comes below.

\section{Lemma 4.2 (Convergence of the cumulants of RWCRE).}

$$
\lim _{n \rightarrow \infty} \frac{1}{n} \log E_{0}^{\Omega, \tau}\left[e^{\lambda X_{n}}\right]=\sup _{x \in \mathbb{R}}[\lambda x-\mathcal{I}(x)]=\mathcal{I}^{*}(\lambda), \quad \forall \lambda \in \mathbb{R} .
$$

Proof. By (1.26) and the independence between $\omega_{k}$ and $X_{\tau(k-1)}$, we have the following equality in distribution:

$$
\frac{1}{n} \log E_{0}^{\Omega, \tau}\left[e^{\lambda X_{n}}\right] \stackrel{(\mathrm{d})}{=} \frac{1}{n} \sum_{k=1}^{\ell(n)-1} \log E_{0}^{\omega_{k}}\left[e^{\lambda Z_{T_{k}}}\right]+\frac{1}{n} \log E_{0}^{\omega_{\ell(n)}}\left[e^{\lambda Z_{\bar{T}} n}\right] .
$$

Now, let $\mathbb{P}$ be the law induced by the doubly indexed variables $\psi_{n}^{(k)}(\Omega):=\log E_{0}^{\omega_{k}}\left[e^{\lambda Z_{n}}\right]$ under $\mu^{\mathbb{N}}$. Then

$$
\psi_{T_{k}}^{(k)}=\log E_{0}^{\omega_{k}}\left[e^{\lambda Z_{T_{k}}}\right], \quad \psi_{\bar{T}^{n}}^{(\ell(n))}=\log E_{0}^{\omega_{\ell(n)}}\left[e^{\lambda Z_{\bar{T}^{n}}}\right] .
$$

Assumptions (A1) and (A2) of Theorem 1.12 are readily satisfied and, by (1.33), (1.29) holds with $L=\mathcal{I}^{*}(\lambda)$ and $\delta>1$. The claim follows from Theorem 1.12.

\subsubsection{Existence of the rate function}

We show that $\mu^{\mathbb{N}}$-a.e. $\Omega$, for every $x \in[-1,1]$,

$$
\mathcal{I}(x) \leq \liminf _{n \rightarrow \infty}-\frac{1}{n} P_{0}^{\Omega, \tau}\left(X_{n}=[[n x]]\right) \leq \limsup _{n \rightarrow \infty}-\frac{1}{n} P_{0}^{\Omega, \tau}\left(X_{n}=[[n x]]\right) \leq \mathcal{I}(x) .
$$

Upper bound. By (1.26) and the Chapman-Kolmogorov equation, we can estimate

$$
\begin{gathered}
P_{0}^{\Omega, \tau}\left(X_{n}=[[n x]]\right) \geq P_{0}^{\omega_{1}}\left(Z_{T_{1}}=[[\tau(1) x]]\right) \times P_{[[\tau(1) x]]}^{\omega_{2}}\left(Z_{T_{2}}=[[\tau(2) x]]\right) \\
\times \cdots \times P_{[[\tau(\ell(n)-1) x]]}^{\omega_{\ell(n)}}\left(Z_{\bar{T}^{n}}=[[n x]]\right) .
\end{gathered}
$$

Define the spatial-shift operator $\left(\theta_{z} \omega\right)(y):=\omega(z+y)$ and note that

$$
-\log P_{[[\tau(k) x]]}^{\omega_{(k+1)}}\left(Z_{n}=[[(\tau(k)+n) x]]\right)=-\log P_{0}^{\theta_{[[\tau(k) x]]} \omega_{k+1}}\left(Z_{n}=[[n x]]\right) .
$$

By the spatial-shift invariance of $\omega$ under $\mu$, we have the following equality in distribution:

$$
\psi_{n}^{(k)}:=-\frac{1}{n} \log P_{0}^{\theta_{[[\tau(k) x]]} \omega_{k+1}}\left(Z_{n}=[[n x]]\right) \stackrel{(\mathrm{d})}{=}-\frac{1}{n} \log P_{0}^{\omega_{k}}\left(Z_{n}=[[n x]]\right) .
$$


We next want to show that Theorem 1.12 can be applied to the above defined doubleindexed sequence. Condition (A1) follows from the fact that, under $\mu^{\mathbb{N}},\left(\psi_{n}^{(k)}\right)_{n \in \mathbb{N}}$ and $\left(\psi_{n}^{\left(k^{\prime}\right)}\right)_{n \in \mathbb{N}}$ are independent. Uniform ellipticity implies (A2) and Theorem 1.12 implies (1.29) with $L=\mathcal{I}(x)$ and $\delta>1$. Therefore, by (1.30),

$$
\limsup _{n \rightarrow \infty}-\frac{1}{n} \log P_{0}^{\Omega, \tau}\left(X_{n}=[[n x]]\right) \leq \mathcal{I}(x) .
$$

This proves the desired upper bound.

Lower bound. The first inequality in (4.8) follows by an exponential Markov inequality in combination with Lemma 4.2. First note that

$$
P_{0}^{\Omega, \tau}\left(X_{n}=[[n x]]\right) \leq \min \left\{P_{0}^{\Omega, \tau}\left(X_{n} \geq[[n x]]\right), P_{0}^{\Omega, \tau}\left(X_{n} \leq[[n x]]\right)\right\} .
$$

The Chernoff bound yields

$$
\begin{array}{ll}
\theta>0: & P_{0}^{\Omega, \tau}\left(X_{n} \geq[[n x]]\right)=P_{0}^{\Omega, \tau}\left(e^{\theta X_{n}} \geq e^{\theta[[n x]]}\right) \leq \frac{1}{e^{\theta[[n x]]}} E_{0}^{\Omega, \tau}\left[e^{\theta X_{n}}\right], \\
\theta<0: & P_{0}^{\Omega, \tau}\left(X_{n} \leq[[n x]]\right)=P_{0}^{\Omega, \tau}\left(e^{\theta X_{n}} \geq e^{\theta[[n x]]}\right) \leq \frac{1}{e^{\theta[[n x]]}} E_{0}^{\Omega, \tau}\left[e^{\theta X_{n}}\right] .
\end{array}
$$

Taking $-\frac{1}{n} \log$ on both sides of (4.14), we get

$$
-\frac{1}{n} \log P_{0}^{\Omega, \tau}\left(X_{n} \geq[[n x]]\right) \geq-\frac{1}{n} \log \frac{1}{e^{\theta[[n x]]}} E_{0}^{\Omega, \tau}\left[e^{\theta X_{n}}\right]=\theta \frac{[[n x]]}{n}-\frac{1}{n} \log E_{0}^{\Omega, \tau}\left[e^{\theta X_{n}}\right],
$$

and from Lemma 4.2 it follows that

$$
\liminf _{n \rightarrow \infty}-\frac{1}{n} \log P_{0}^{\Omega, \tau}\left(X_{n} \geq[[n x]]\right) \geq \theta x-\mathcal{I}^{*}(\theta) .
$$

We can argue analogously for (4.15). In conclusion, the lower bound follows since

$$
\begin{aligned}
& x>0 \Longrightarrow \sup _{\theta>0}\left(\theta x-\mathcal{I}_{\mu}^{*}(\theta)\right)=\mathcal{I}(x), \\
& x<0 \Longrightarrow \sup _{\theta<0}\left(\theta x-\mathcal{I}_{\mu}^{*}(\theta)\right)=\mathcal{I}(x) .
\end{aligned}
$$

\subsubsection{LDP given existence of the rate function}

The proof is classical and we closely follow the argument in [5, p. 14-15]. We need to prove that $\mu^{\mathbb{N}}$ almost surely, for any open set $\mathcal{O} \subset \mathbb{R}$,

$$
\liminf _{n \rightarrow \infty} \frac{1}{n} P_{0}^{\Omega, \tau}\left(\frac{X_{n}}{n} \in \mathcal{O}\right) \geq-\inf _{x \in \mathcal{O}} \mathcal{I}(x)
$$

and, for any closed set $\mathcal{C} \subset \mathbb{R}$,

$$
\limsup _{n \rightarrow \infty} \frac{1}{n} P_{0}^{\Omega, \tau}\left(\frac{X_{n}}{n} \in \mathcal{C}\right) \leq-\inf _{x \in \mathcal{C}} \mathcal{I}(x) .
$$

To check (4.20), since $\mathcal{O}$ is an open set for any $x \in \mathcal{O}$, we have $\frac{[[n x]]}{n} \in \mathcal{O}$ for sufficiently large $n$. Therefore, by (4.8),

$$
\begin{aligned}
\liminf _{n \rightarrow \infty} \frac{1}{n} \log P_{0}^{\Omega, \tau}\left(\frac{X_{n}}{n} \in \mathcal{O}\right) & =-\limsup _{n \rightarrow \infty}-\frac{1}{n} \log P_{0}^{\Omega, \tau}\left(\frac{X_{n}}{n} \in \mathcal{O}\right) \\
& \geq-\limsup _{n \rightarrow \infty}-\frac{1}{n} \log P_{0}^{\Omega, \tau}\left(X_{n}=[[n x]]\right) \\
& =-\mathcal{I}(x)
\end{aligned}
$$


and since $x \in \mathcal{O}$ is arbitrary, (4.20) follows. To obtain (4.21), note that, because $\left(\frac{X_{n}}{n} \in \mathcal{C}\right)=\left(\frac{X_{n}}{n} \in \mathcal{C} \cap[-1,1]\right)$ and

$$
\#\left\{y \in \mathbb{Z}: \frac{y}{n} \in \mathcal{C} \cap[-1,1]\right\} \leq 2 n+1,
$$

it follows that

$$
P_{0}^{\Omega, \tau}\left(\frac{X_{n}}{n} \in \mathcal{C}\right) \leq(2 n+1) \sup _{x \in \mathcal{C}} P_{0}^{\Omega, \tau}\left(X_{n}=[[n x]]\right),
$$

and since $\frac{\log n}{n} \rightarrow 0$, we get that

$$
\limsup _{n \rightarrow \infty} \frac{1}{n} \log P_{0}^{\Omega, \tau}\left(\frac{X_{n}}{n} \in \mathcal{C}\right)=\limsup _{n \rightarrow \infty} \sup _{x \in \mathcal{C}} \frac{1}{n} \log P_{0}^{\Omega, \tau}\left(X_{n}=[[n x]]\right) .
$$

To conclude the claim it suffices to show that, for any $\varepsilon>0$,

$$
\limsup _{n \rightarrow \infty} \sup _{x \in \mathcal{C}} \frac{1}{n} \log P_{0}^{\Omega, \tau}\left(X_{n}=[[n x]]\right) \leq-\inf _{x \in \mathcal{C}} \mathcal{I}(x)+\varepsilon .
$$

To see the latter, we argue by contradiction. Indeed, assuming that (4.26) is false, we can find a sequence of points $z_{n_{k}} \in \mathcal{C} \cap[-1,1]$, converging to $z \in \mathcal{C} \cap[-1,1]$, such that

$$
\frac{1}{n_{k}} \log P_{0}^{\Omega, \tau}\left(X_{n_{k}}=\left[\left[n_{k} z_{n_{k}}\right]\right]\right)>-\inf _{x \in \mathcal{C}} \mathcal{I}(x)+\varepsilon .
$$

By (4.8) and (4.3) we obtain that

$$
\begin{aligned}
-\mathcal{I}(z) & =-\lim _{k \rightarrow \infty} \frac{1}{n_{k}^{\prime}} \log P_{0}^{\Omega, \tau}\left(X_{n_{k}^{\prime}}=\left[\left[n_{k}^{\prime} z\right]\right]\right) \\
& \geq-\lim _{k \rightarrow \infty} \frac{1}{n_{k}} \log P_{0}^{\Omega, \tau}\left(X_{n_{k}}=\left[\left[n_{k} z_{n_{k}}\right]\right]\right)>-\inf _{x \in \mathcal{C}} \mathcal{I}(x)+\varepsilon,
\end{aligned}
$$

which gives the desired contradiction and concludes the proof of Theorem 1.11.

\section{References}

[1] S. Ahn and J. Peterson, Quenched central limit theorem rates of convergence for onedimensional random walks in random environments, Bernoulli 25 (2019), no. 2, 1386-1411. MR-3920376

[2] L. Avena, O. Blondel, and A. Faggionato, Analysis of random walks in dynamic random environments via $L^{2}$-perturbations, Stoch. Proc. Appl. 128 (2018), no. 10, 3490 - 3530. MR-3849817

[3] L. Avena and F. den Hollander, Random walks in cooling random environments, arXiv e-prints (2017), arXiv:1610.00641.

[4] C. Boldrighini, R. Minlos, and A. Pellegrinotti, Random walks in quenched i.i.d. space-time random environment are always a.s. diffusive, Probab. Theory Relat. Fields 129 (2004), no. 1, 133-156. MR-2052866

[5] D. Campos, A. Drewitz, A. Ramírez, F. Rassoul-Agha, and T. Seppäläinen, Level 1 quenched large deviation principle for random walk in dynamic, Bull. Inst. Math. Acad. Sin. 8 (2013), 1-29. MR-3097414

[6] F. Comets, N. Gantert, and O. Zeitouni, Quenched, annealed and functional large deviations for one-dimensional random walk in random environment, Probab. Theory Relat. Fields 118 (2000), no. 1, 65-114. MR-1785454

[7] A. Dembo, Y. Peres, and O. Zeitouni, Tail estimates for one-dimensional random walk in random environment, Commun. in Math. Phys. 181 (1996), no. 3, 667-683. MR-1414305

[8] A. Greven and F. den Hollander, Large deviations for a random walk in random environment, Ann. Probab. 22 (1994), no. 3, 1381-1428. MR-1303649 
RWCRE: ergodic limits and concentration inequalities

[9] F. den Hollander, Large deviations, Fields Institute monographs, American Mathematical Society, 2000. MR-1739680

[10] H. Kesten, The limit distribution of Sinai's random walk in random environment, Phys. A 138 (1986), 299-309. MR-0865247

[11] H. Kesten, M. Kozlov, F. Spitzer, A limit law for random walk in a random environment, Comp. Math. 30 (1975), no. 2, 145-168. MR-0380998

[12] T. Liggett, An improved subadditive ergodic theorem, Ann. Probab. 13 (1985), no. 4, 12791285. MR-0806224

[13] P. Révész, The Laws of Large Numbers, Probability and Mathematical Statistics, Academic Press, 1968. MR-0245079

[14] Y. Sinai, The limiting behavior of a one-dimensional random walk in a random medium, Theory Probab. Appl. 27 (1982), no. 2, 256-268. MR-0657919

[15] F. Solomon, Random walks in a random environment, Ann. Probab. 3 (1975), no. 1, 1-31. MR-0362503

[16] O. Zeitouni, Lectures on probability theory and statistics, Lecture Notes in Mathematics, vol. 1837, 189-312, 2004. MR-2071629

Acknowledgments. The research in this paper was supported through NWO Gravitation Grant NETWORKS-024.002.003. 


\section{Electronic Journal of Probability Electronic Communications in Probability}

\section{Advantages of publishing in EJP-ECP}

- Very high standards

- Free for authors, free for readers

- Quick publication (no backlog)

- Secure publication $\left(\mathrm{LOCKSS}^{1}\right)$

- Easy interface (EJMS²)

\section{Economical model of EJP-ECP}

- Non profit, sponsored by $\mathrm{IMS}^{3}, \mathrm{BS}^{4}$, ProjectEuclid ${ }^{5}$

- Purely electronic

\section{Help keep the journal free and vigorous}

- Donate to the IMS open access fund ${ }^{6}$ (click here to donate!)

- Submit your best articles to EJP-ECP

- Choose EJP-ECP over for-profit journals

\footnotetext{
${ }^{1}$ LOCKSS: Lots of Copies Keep Stuff Safe http://www. lockss.org/

${ }^{2}$ EJMS: Electronic Journal Management System http://www.vtex.lt/en/ejms.html

${ }^{3}$ IMS: Institute of Mathematical Statistics http://www.imstat.org/

${ }^{4}$ BS: Bernoulli Society http://www. bernoulli-society.org/

${ }^{5}$ Project Euclid: https://projecteuclid.org/

${ }^{6}$ IMS Open Access Fund: http://www.imstat.org/publications/open.htm
} 